\title{
Retrieval of hydrometeors from microwave radiances with a polarized radiative transfer model
}

\author{
C Balaji ${ }^{1, *}$, M Deiveegan ${ }^{1}, \mathrm{~S}_{\text {P Venkateshan }}{ }^{1}$, R M Gairola ${ }^{2}$, \\ A SARKAR ${ }^{2}$ and V K AgARWAL ${ }^{2}$ \\ ${ }^{1}$ Department of Mechanical Engineering, Indian Institute of Technology Madras, Chennai, India. \\ ${ }^{2}$ Meteorology and Oceanography Group, Space Applications Centre, ISRO Ahmedabad, India. \\ *e-mail: balaji@iitm.ac.in
}

This paper reports the results of a Bayesian-based algorithm for the retrieval of hydrometeors from microwave satellite radiances. The retrieval technique proposed makes use of an indigenously developed polarized radiative transfer (RT) model that drives a data driven optimization engine (Bayesian) to perform retrievals of rain and other hydrometeors in a multi-layer, plane parallel raining atmosphere. For the sake of completeness and for the purposes of comparison, retrievals with Artificial Neural Networks (ANN) have also been done. Retrievals have been done first with a simplified two-layer atmosphere, where assumed values of hydrometeors are given to the forward model and these are taken as 'measured radiances'. The efficacy of the two retrieval strategies is then tested for this case in order to establish accuracy and speed. The highlight of the work is however, the case study wherein a tropical storm in the Bay of Bengal is taken up, to critically examine the performance of the retrieval algorithm for an extreme event wherein a 14-layer realistic, raining atmosphere has been considered and retrievals are done against Tropical Rainfall Measuring Mission (TRMM) measured radiances. The key novelties of the work are:

- inclusion of polarization from both hydrometeors and oceans in the RT model, and

- populating the database involving atmospheric profiles $v s$. simulated radiances by profiles of similar rain events in the past.

In this work, the database was populated with TRMM retrieved profiles for tropical cyclones that occurred earlier in the area of interest (Indian Ocean), rather than with the Goddard Cloud Ensemble profiles. The use of (i) polarization in the forward model and (ii) creation of an a priori database for the retrieval denote the significant departure from the current state-of-the-art in the area.

\section{Introduction}

In the atmosphere, quantitative assessment of rainfall is needed to improve the understanding of global weather. Since $70 \%$ of the earth is covered with water, land-based techniques of rainfall estimation (example: rain gauges) are not sufficient for global rainfall estimation. Hence, one uses satellite remote sensing for global estimation of rainfall.
Based on sensor frequency, remote sensing is divided into three main fields:

(i) visual remote sensing, which covers wavelengths from approximately 0.3 to $0.7 \mu \mathrm{m}$,

(ii) infrared remote sensing, which covers wavelengths from approximately 0.7 to $1000 \mu \mathrm{m}$, and

(iii) microwave remote sensing, which covers wavelengths from approximately $1 \mathrm{~mm}$ to $30 \mathrm{~cm}$.

Keywords. Radiative transfer model; plane parallel atmosphere; polarization; hydrometeors; retrievals; microwave radiances. 
Since visual and infrared radiation cannot penetrate the clouds, the presence of clouds affects these measurements. At any given time, approximately $40 \%$ of the earth is covered with clouds. Microwave remote sensing is a valuable way of measuring geophysical parameters because it can partially penetrate the clouds, and microwave radiometers are able to measure blackbody emissions day or night and are nearly independent of weather conditions. Compared to radars, radiometers are cost-effective and require less electrical power, which is important in space-borne applications.

Microwave remote sensing problems are of the general class of inverse problems, where the sensor gives a measurement vector from which the parameter vector needs to be inferred. Several inversion techniques have been proposed and applied in the last three decades for estimating precipitation profiles. A detailed review of literature shows that the Bayesian techniques and artificial neural network have proven potential and flexibility (Evans and Stephens 1995; Marzano et al 1999).

Physics-based techniques provide solutions to two classes of problems, the forward problem and the inverse problem. The forward problem consists of the generation of a database, in which the simulated brightness temperatures that would be measured by a space-borne radiometer are associated with various rain cloud structures. The output of the forward model is the creation of database which is significant for retrievals. In the inverse problem, the database is used to train the retrieval algorithm that minimizes the difference between simulated and measured brightness temperatures. The output of the retrieval algorithm is the vertical profile of precipitation and related parameters.

\section{Methodology}

The remote measurement of atmospheric rain and related parameters by microwave radiometers is a typical inverse problem and can be mathematically stated as follows:

$$
y=F(x),
$$

where the vector $y$ is the measurement vector, e.g., the discrete values of the brightness temperatures at different frequencies and polarization, whereas $x$ is the parameter vector to be measured, e.g., discrete values of rain, cloud liquid water, precipitating ice and cloud ice at different altitude. Here, one is interested in $x$ but is only able to measure $y$. The forward model $F$ has to estimate the measurement vector $y$ for a given parameter vector $x$.
There are methods to estimate $x$ if $y$ is known but they all require the forward model $F$ to be known. A direct inversion of equation (1), to obtain $x$ directly given $y$ is seldom possible, as the inverse problem is typically ill-posed or underconstrained, outside of the errors associated with measurements and the forward model solution. Stated explicitly, for a typical microwave remote sensing problem, $x$ typically represents the vertical structure of rain, ice and clouds and may involve around 50-100 layer averaged parameters, depending on the number of vertical layers into which the atmosphere is divided for computational purposes. The $y$ values represent the microwave radiances which may vary from 5 to 10 depending on the number of channels used in the remote sensing instrument. Hence, in principle there could be several combinations of parameters constituting $x$ that can give rise to the same $y$ making the retrieval process extremely formidable.

The present work deals with remote measurements on satellites using passive microwave techniques with a view to develop an in-house, indigenous retrieval algorithm for use in the proposed Indo-French climate research satellite MEGHA-TROPIQUES due to be launched in 2009-2010 (see Srinivasan 2006). The microwave radiances are measured with a microwave radiometer and a mathematical model known as the Radiative Transfer Model is developed to account for the radiance collected by the radiometer antenna. The theory of radiative transfer in participating medium is essential to interpret the measured radiances to infer the physical properties of media that produced them. The process of inferring constituents and its distributions from observations called 'retrieval' is mathematically referred to as an inverse problem, and is typically ill-posed, as already mentioned.

The inverse problem actually uses the forward model as $z=F(x)$ for the relation between the simulated measurement vector $z$ and the assumed state vector $x$ of constituent parameters to be retrieved; $F$ is the forward model, which encompasses our understanding of the physics behind the measurement process. The retrieval involves the solution of the formal inverse problem $x=F^{-1}(z)$. The objective function $(R(x))$ to be minimized is given by,

$$
R(x)=\sum_{k=1}^{N_{s}} \sum_{i=1}^{N_{o}}\left[y_{k, i}-z_{k, i}(x)\right]^{2},
$$

where $N_{s}$ is number of frequency bands, $N_{o}$ is the number of observations, $y$ and $z$ are the measured and simulated vectors, respectively. Multiple 


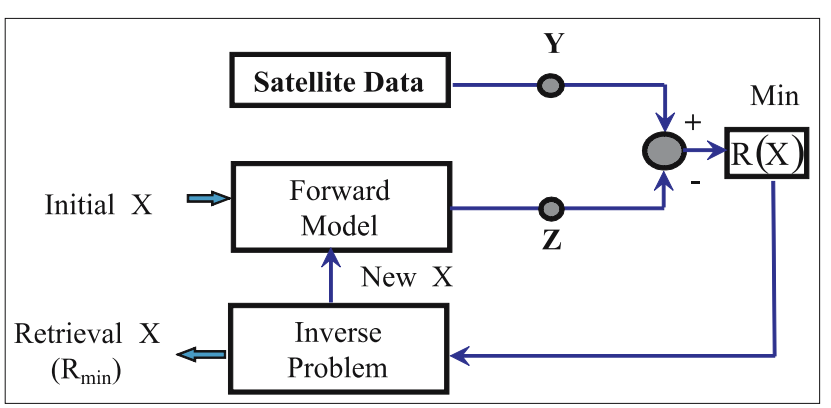

Figure 1. Inverse methodology adopted in the present work.

channels are used to increase the information content in the problem with a view to reduce the ill-posedness. The tropical rainfall measurement mission (TRMM) of NASA that is operational from 1997 onwards has the following microwave channels $(10.65,19.35,37,85.5 \mathrm{GHz}$ dual polarization and $22.235 \mathrm{GHz}$ channel vertical polarization) in its microwave imager known as the TRMM microwave imager (TMI). The proposed Indo-French climate research satellite MEGHATROPIQUES has similar channels except for the $10 \mathrm{GHz}$ channel of the TMI imager that will be replaced by the $157 \mathrm{GHz}$ ice scattering channel.

Figure 1 gives a schematic of the inverse methodology adopted in the present work. The retrieval scheme that links the brightness temperature to physical parameters (precipitable water, cloud liquid water, cloud ice, precipitating ice) is regularly set up as an optimization problem that utilizes forward model simulations. Numerical models are used to compute the microwave radiation within a well defined atmosphere. The inversion of such calculations needs a retrieval algorithm. In view of the above, it is clear that the quality of the retrieval algorithm is directly connected to:

- the quality of the forward model,

- the parameter estimation algorithm used for the retrieval, and

- the prior knowledge we have about the parameters or the state vector.

\subsection{The forward model}

The retrieval process begins with the development of a polarized microwave model that can compute the radiances leaving the top of the atmosphere at various microwave frequencies for a given atmospheric state. The first step is to generate an atmosphere with realistic physical parameters. The second step is to convert all the atmospheric parameters or hydrometeors (rain, cloud liquid water, cloud ice, precipitating ice) into radiative interaction parameters. The interaction parameters describe the interaction between a medium and an electromagnetic wave passing through it. These are:

- the extinction coefficients,

- single scattering albedo,

- the phase matrix for the atmosphere,

- the emissivity matrix, and

- the bidirectional reflection matrix for the ocean.

The radiative transfer equation is then solved using these interaction parameters in order to determine the top of the atmosphere (TOA) radiances.

The forward radiative transfer model is divided into 3 modules (figure 2):

- generation of the physical and interaction parameters for the atmosphere,

- generation of the physical and interaction parameters for the ocean surface, and

- solution of the vector radiative transfer equations.

The generation of the atmospheric and the ocean surface parameters is very highly involved wherein the theory of scattering is used in conjunction with the assumption of distribution of the droplet sizes for various hydrometeors. A full discussion on the forward model is available in an earlier article by the first three authors (Deiveegan et al 2008).

\subsubsection{Solution of vector radiative transfer equations}

A crucial step in the development of polarized microwave radiative transport model is the solution of the vector radiative transfer equation. In the present work, the polarized adding and doubling method was used to solve the vector radiative transfer equations. The radiative interaction parameters required are calculated from the above-mentioned formulation. The interaction parameters like extinction, scattering matrices, phase matrix for atmosphere and reflection matrix and emission matrix for surfaces are given as input. A solution of vector radiative transfer equations gives the brightness temperatures for the vertical and horizontal polarizations at a given frequency. Figure 3 shows a schematic of the third step involved in the solution of vector radiative transfer equations.

The radiative transfer model gives the simulated brightness temperatures (or radiance) for each viewing angle and frequency, for a downward looking space borne radiometer. The radiative interaction parameters for the medium (extinction, scattering albedo and phase matrix) and the surface (bidirectional reflection matrix, emission matrix) are given as inputs for the radiative transfer calculations. A detailed flow diagram indicating 


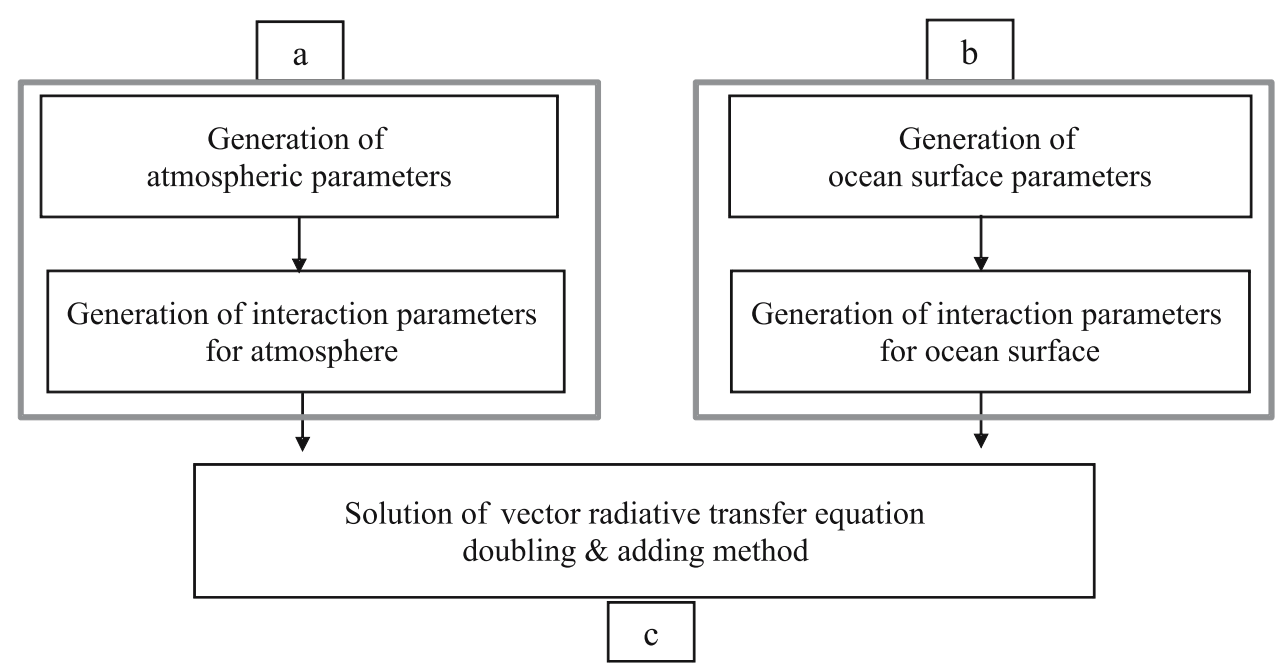

Figure 2. Three important modules of a polarized microwave radiative transfer model.

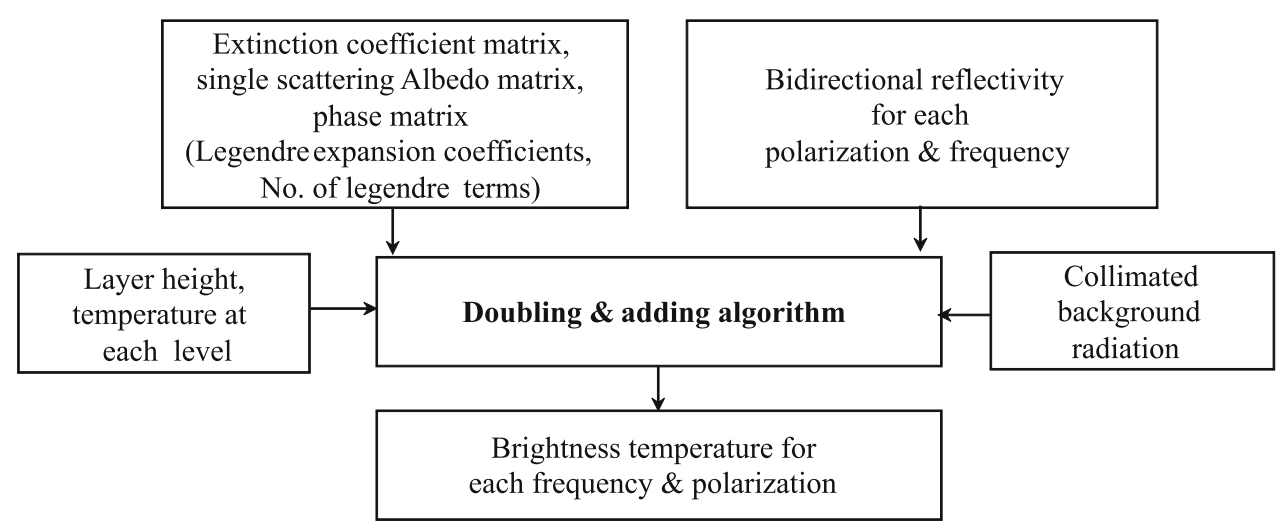

Figure 3. Schematic illustrating calculation of top of the atmosphere radiances using the adding and doubling method.

the various steps employed in the forward model is shown in figure 3. Following, Evans and Stephens (1991), the monochromatic plane parallel polarized radiative transfer equation for randomly oriented particles can be written as:

$$
\begin{aligned}
& \mu \frac{d I}{d \tau}(\tau, \mu, \phi)=-I(\tau, \mu, \phi)+\frac{\tilde{\omega}}{4 \pi} \\
& \quad \times \int_{0}^{2 \pi} \int_{-1}^{1} M(\mu, \phi ; \tilde{\mu}, \tilde{\phi}) d \tilde{\mu} d \tilde{\phi}+(1-\tilde{\omega}) B(T)\left[\begin{array}{l}
1 \\
0 \\
0 \\
0
\end{array}\right] .
\end{aligned}
$$

Here $I$ is the diffuse radiance field expressed as the vector of four Stokes parameters $(I, Q$, $U, V), M$ is the $4 \times 4$ scattering (or Mueller) matrix, $B(T)$ is the Planck blackbody function, $\tilde{\omega}$ the single scattering albedo, $\tau$ the optical depth, $\mu$ the cosine of the zenith angle $\theta$ and $\phi$ the azimuth angle. The coordinate system is such that $\tau$ increases downwards and $\mu$ is positive for the lower hemisphere. The angular variation of radiation is written as a Fourier series in the azimuth direction and by discretization in the zenith angle using numerical Gaussian quadrature. The radiance at any position inside the atmosphere is represented by the following: Stokes parameters, quadrature zenith angles and the Fourier azimuth modes. The radiance field is computed via the doubling-adding algorithm. Detailed validations of the forward model are again available in Deiveegan et al (2008).

\subsection{Retrieval algorithms}

The primary objective of the present work is to present a Bayesian framework with a new method of populating the database. However, for purposes of comparing the accuracy of the retrievals another data driven, stochastic optimization technique, namely artificial neural networks (ANN) was also used. 


\subsubsection{Bayesian retrieval algorithm}

A Bayesian probabilistic framework allows incorporation of various informational sources in a rigorous and physically meaningful way. The Bayesian framework allows incorporation of numerous information sources, providing additional constraints for ill-posed inverse problems. One possible approach to Bayesian retrievals is the use of an offline database of pre-calculated simulated vectors for many possible state vectors, and integrating over the points in the database with the Bayes' theorem. Bayesian inversion methods formally add prior information to that provided by the measurements to obtain a well posed retrieval. The Bayes' theorem can be stated mathematically for the retrieval problem as:

$$
p_{\text {post }}(x \mid y)=\frac{p_{f}(y \mid x) p_{p r}(x)}{\int p_{f}(y \mid x) p_{p r}(x) d x},
$$

where $x$ represents the state vector and $y$ represents the vector of observations. $p_{p r}(x)$ is the prior probability density function (PDF) of the state $x, p_{f}(y \mid x)$ is the conditional probability density function of the measurements given the state vector, and $p_{\text {post }}(x \mid y)$ is the posterior probability density function (PPDF) of the state vector. The prior PDF represents our knowledge of the parameters.

Once the PPDF of the state vector is obtained, the retrieved parameter $x_{\text {ret }}$ is calculated by integrating over the posterior PDF to determine the mean state, which is actually the expectation of $x$ :

$$
x_{\mathrm{ret}}=\frac{\int x p_{f}(y \mid x) p_{p r}(x) d x}{\int p_{f}(y \mid x) p_{p r}(x) d x} .
$$

This is a Monte Carlo integration because the database points are chosen randomly. In practice, this integral is replaced by a sum over the cases in the database. One advantage of the Bayesian framework is that the uncertainties in retrieved parameters are naturally defined by the variance of the posterior PDF:

$$
\sigma_{x}^{2}=\frac{\int\left(x-x_{\mathrm{ret}}\right)^{2} p_{f}(y \mid x) p_{p r}(x) d x}{\int p_{f}(y \mid x) p_{p r}(x) d x},
$$

where $\sigma_{x}$ is the one sigma error bar in $x$ and $x_{\text {ret }}$ is the mean $x$ of the posterior PDF. The forward PDFs of measurement vector are assumed to be normally distributed about the simulated vector for each observation as follows:

$$
p_{f}(y \mid x)=\prod_{j=1}^{N_{\mathrm{ch}}} \frac{1}{\sqrt{2 \pi \sigma_{j}^{2}}} \exp \left[-\frac{\left(y_{j}-Z_{j}(x)\right)^{2}}{2 \sigma_{j}^{2}}\right],
$$

where $y_{j}$ is the $j$ 'th channel in the measurement vector, $Z_{j}(x)$ is the radiative transfer simulation for channel $j, N_{\mathrm{ch}}$ is the number of channels, and $\sigma_{j}$ is the standard deviation for channel $j$. This formulation assumes that the uncertainty in each channel is independent of the other channels, which is a good assumption for random measurement noise, but may not be good for representing the radiative transfer modelling error. It is assumed that the uncertainty $\sigma_{j}$ is due to the measurement errors. The conditional distribution is close to zero if the measurement vector is far from the observed one and hence the Bayesian algorithm interpolates between those points in the database that are reasonable matches to the observations.

The prior probability distribution is a way of introducing other known information about the parameters, and addition of a priori information can serve as a constraint. In addition, the prior distribution gives a good indication of how to choose the random cases for the database. Upon using a pre-calculated database where $p_{p r}(x)=1$, the Monte Carlo integration simplifies to:

$$
x_{\mathrm{ret}}=\frac{\sum_{i} x_{i} p_{f}\left(y \mid x_{i}\right)}{\sum_{i} p_{f}\left(y \mid x_{i}\right)} .
$$

Equation (8) follows from the application of equation (7) for $p_{f}(y / x)$ in equation (6). Additionally, the denominator in equation (6) is made 1 as it is only a normalizing factor and the integral is replaced by a summation as the least square error (i.e., the sum of the square of the difference between the simulated and satellite measured radiances over all the channels) is done at a discrete number of points. The discrete number of points refers to the discrete number of sample hydrometeor profiles that are tested for ultimately evaluating the 'expected value' in a probabilistic sense. Hence, the sums in the Bayesian retrieval algorithm actually span all points in the database. However, including all the database points wastes computation time calculating the conditional PDF for points where the PDF is effectively zero (i.e., where the measurement is far from that of the database case). The conditional PDF is the product of exponentials, and thus is also the exponential of $\chi^{2}$ :

$$
x_{\mathrm{ret}}=\frac{\sum_{i} x_{i} \exp \left[-\frac{1}{2} \chi_{i}^{2}\right]}{\sum_{i} \exp \left[-\frac{1}{2} \chi_{i}^{2}\right]}, \quad x_{i} \text { from } p_{p r}(x),
$$


where $\chi^{2}$ is a measure of the square of the difference between the measurement vector and the simulated vector from the database.

$$
\chi^{2}=\sum_{i=1}^{N_{\mathrm{ch}}}\left(\frac{y_{i}-Z_{i}}{\sigma_{s t, i}}\right)^{2} .
$$

One way to speed up the Bayes integration is to stop the $\chi^{2}$ summation and not calculate the posterior PDF when the $\chi^{2}$ exceeds a maximum user specified value, $\chi^{2}>\chi_{\max }^{2}$. In the present work $\chi_{\max }^{2}=30$ is used.

\subsubsection{Artificial neural network (ANN)}

This section describes the procedure involved in using artificial neural network as a retrieval algorithm to estimate the precipitation and related parameters from microwave remote sensing data.

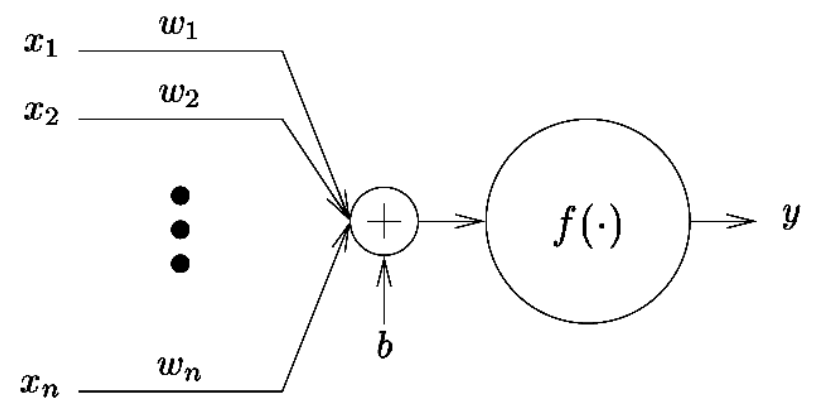

Figure 4. The structure of a perceptron.
Neural network: Neural nets are computational structures that were developed to mimic the way biological neural nets learn from their environment and are useful for pattern recognition and classification. Neural nets can be used to learn and compute functions for which the relationship between inputs and outputs are unknown or computationally complex. There are a variety of neural nets (Lippmann 1987) such as feed forward neural nets (multilayer perceptrons), Kohonen self-organizing feature maps, and Hopfield nets. In this study, the feed forward neural net has been employed.

Structure of neural network: The basic structural element of feed forward neural nets is called a perceptron. It computes a function of the weighted sum of inputs and a bias, as shown in figure 4:

$$
y=f\left(\sum_{i=1}^{n} w_{i} x_{i}+b\right) \text {, }
$$

where $x_{i}$ is the $i$ th input, $w_{i}$ is the weight associated with the $i$ th input, $b$ is the bias, $f$ is the transfer function of the perceptron, and $y$ is the output.

Perceptrons can be combined to form a multilayer network as shown in figure 5. In figure $5, x_{i}$ is the $i$ th input, $n$ is the number of inputs, $w_{i j}$ is the weight associated with the connection from the $i$ th input to the $j$ th node in the hidden layer, $b_{i}$ is the bias of the $i$ th node, $m$ is the number of nodes in the hidden layer, $f$ is the transfer function of the

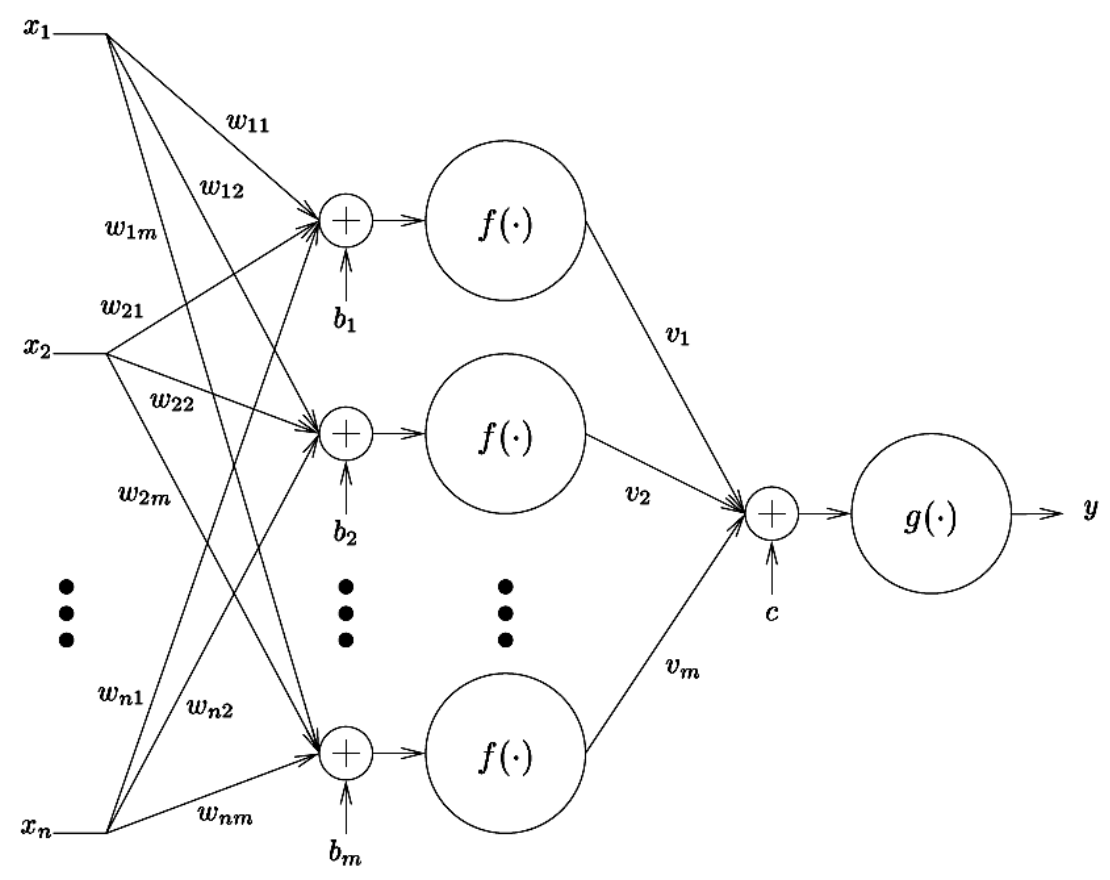

Figure 5. A two-layer feed forward neural net with one output node. 


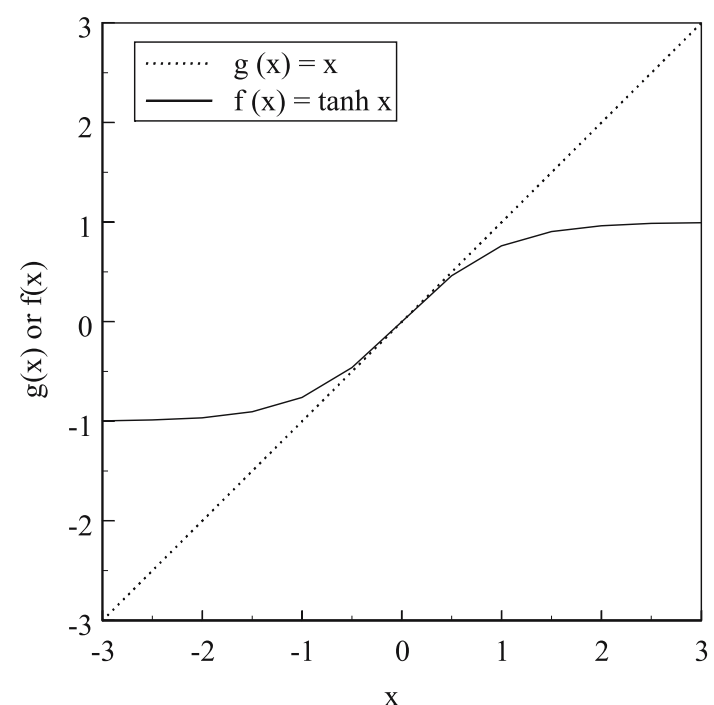

Figure 6. Neural network transfer functions.

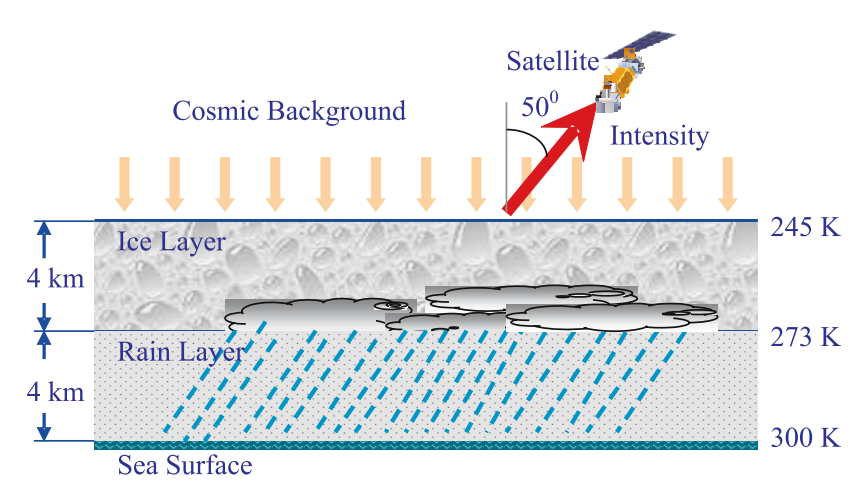

Figure 7. A simple two-layer atmosphere model.

perceptrons in the hidden layer, $w_{i}$ is the weight between the $i$ th node and the output node, $c$ is the bias of the output node, $g$ is the transfer function of the output node, and $y$ is the output. Then,

$$
y=g\left(\sum_{j=1}^{m} v_{j} f\left(\sum_{i=1}^{n} w_{i} x_{i}+b_{j}\right)+c\right) .
$$

In the present work, $f$ and $g$ are defined as follows:

$$
\begin{aligned}
& f(x)=\tanh x=\frac{e^{x}-e^{-x}}{e^{x}+e^{-x}}, \\
& g(x)=x
\end{aligned}
$$

The function $\tanh x$ is approximately linear in the range $-0.6 \leq x \leq 0.6$, and approaches 1 as $x$ goes to 1 and -1 as $x$ goes to -1 and so it has a nonlinearity that is not too complex (figure 6).
Training the neural network The neural nets for this study were trained using the LevenbergMarquardt training algorithm. Marquardt developed an efficient algorithm (Hagan and Menhaj 1994) for nonlinear least-squares parameter estimation (Marquardt 1963). Hagan and Menhaj (1994) incorporated this technique into a back propagation training algorithm for feed forward neural nets.

The weights of the neural net were initialized using the Nguyen-Widrow method in order to facilitate convergence of the neural net weights during training (Nguyen and Widrow 1990). The vectors used to train and evaluate the neural nets were divided into three disjoint sets:

- The training set, the set used to determine how the weights of the neural net are to be adjusted during the training.

- The validation set, the set used to determine when the training should stop.

- The testing set, the set used to evaluate the resulting neural net.

In the training phase, many pairs of inputs and outputs are shown to the network and the weights within the network are adjusted until the network produces the desired output. In the simulation phase, the training algorithm is deactivated, and the network merely computes the output based on the given inputs.

\subsection{Retrieval in a two-layer atmosphere}

In this exercise, for a simplified two-layer atmosphere the sea surface albedo, rain rate and ice content are estimated simultaneously by inverse analysis, from the knowledge of measured brightness temperatures based on vertical and horizontal polarizations. Measured brightness temperatures are simulated by adding random errors to the brightness temperatures computed from the forward problem. To assess the accuracy of predictions, a statistical analysis is made to establish error bars for the inverse solution. To check the performance and accuracy obtained in the retrieval, a comparison is presented between two retrieval methods, viz., Bayesian algorithm and artificial neural network.

\subsubsection{Forward problem for the two-layer atmosphere}

For simplicity, this example considers a two-layer precipitating atmosphere having an ice layer above a rain layer. The lower half of the atmosphere is modelled as a raining layer and the upper half is taken as a layer containing ice particles (figure 7). Absorption by oxygen, water vapour and cloud 
Table 1. Parameters used in the two-layer atmosphere case.

\begin{tabular}{lcc}
\hline Parameter & Range & Unit \\
\hline Reflectivity (albedo) & $0.01-0.8$ & - \\
First layer rain rate & $0.02-49.0$ & $\mathrm{~mm} / \mathrm{h}$ \\
Second layer ice content & $0.01-24.5$ & $\mathrm{~mm} / \mathrm{h}$ \\
\hline
\end{tabular}

liquid is ignored. Vertical hydrometeor profiles, including rain drops and ice particles are specified from 0 to $8 \mathrm{~km}$ in a series of homogenous layers. Table 1 lists the parameters used in the model. The boundary conditions include contribution of downwelling radiation from space $2.7 \mathrm{~K}$ and sea modelled as Lambertian surface at constant surface temperature of $300 \mathrm{~K}$. The relative humidity in this simple model is set at $90 \%$. A viewing angle of $50^{\circ}$ is used. The size distribution is assumed to follow that of Marshall-Palmer with a maximum diameter of $1.0 \mathrm{~cm}$. The Mie theory is used to determine single scattering properties such as extinction coefficient, single scattering albedo and Legendre series coefficients. The forward problem is used to compute, the upwelling radiances at the top of a raining atmosphere at six microwave frequencies $(6.6,10.7,18.0,37.0,85.6,183.0 \mathrm{GHz})$.

\subsubsection{Retrievals}

This example compares retrievals based on the two methods discussed in an earlier section namely, Bayesian algorithm and ANN. Basically these two methods are data driven approaches and hence the generation of database holds the key for both the methods. The distribution of these parameters is the prior information in the retrieval, so it is important that the profiles are realistic and completely cover the possible realistic parameter range. There are two parts in generating the database:

(1) creating the random profile of albedo, rain rates and ice content,

(2) computing the simulated brightness temperatures.

In the present work a database of size 31,000 is generated, out of these only 15,000 datasets are used for ANN training, but 30,000 datasets are used for Bayesian method and 1000 are used for testing.

The parameters obtained through ANN and Bayesian methods are studied by using the brightness temperature values of the 1000 profiles which were not used for training. Table 2 gives the error statistics for both the ANN and Bayesian retrievals for a case with zero measurement error. It may be observed that the PARAmeters such as albedo, first layer rain rate and second layer ice content are estimated accurately. The time consumed on a $4.0 \mathrm{~GB}$ RAM, $3.2 \mathrm{GHz}$ Pentium-4 processor is only $10 \mathrm{~s}$ (ANN) and $30 \mathrm{~s}$ (Bayesian) for retrieving the parameter set of 1000 profiles. Table 3 shows a comparison of the two retrieval strategies for a case with maximum noise in the measurements is $2 \%$.

It can be observed that both algorithms retrieve the parameters very accurately. Parity plots (not

Table 2. Performance of ANN and Bayesian retrievals for 0\% measurement error.

\begin{tabular}{|c|c|c|c|c|c|c|c|c|}
\hline \multirow[b]{2}{*}{ Sl. no. } & \multirow[b]{2}{*}{$\begin{array}{l}\text { Parameter } \\
\text { retrieved }\end{array}$} & \multirow[b]{2}{*}{ Unit } & \multicolumn{3}{|c|}{$\mathrm{ANN}$} & \multicolumn{3}{|c|}{ Bayesian } \\
\hline & & & $\begin{array}{l}\text { Standard } \\
\text { deviation } \\
\text { of } \\
\text { estimate }\end{array}$ & $\begin{array}{c}\text { Correlation } \\
\text { coefficient } \\
(\%)\end{array}$ & $\begin{array}{l}\text { Time } \\
\text { taken } \\
(\mathrm{s})\end{array}$ & $\begin{array}{c}\text { Standard } \\
\text { deviation } \\
\text { of } \\
\text { estimate }\end{array}$ & $\begin{array}{c}\text { Correlation } \\
\text { coefficient } \\
(\%)\end{array}$ & $\begin{array}{l}\text { Time } \\
\text { taken } \\
(\mathrm{s})\end{array}$ \\
\hline 1 & Albedo & - & 0.0046 & 99.9 & 0.01 & 0.016 & 99.8 & 0.03 \\
\hline 2 & 1st layer rain & $\mathrm{mm} / \mathrm{h}$ & 0.59 & 99.9 & 0.01 & 1.36 & 99.5 & 0.03 \\
\hline 3 & 2nd layer ice & $\mathrm{mm} / \mathrm{h}$ & 0.09 & 99.9 & 0.01 & 0.056 & 99.9 & 0.03 \\
\hline
\end{tabular}

Table 3. Performance of ANN and Bayesian retrievals for $2 \%$ measurement error.

\begin{tabular}{|c|c|c|c|c|c|c|c|c|}
\hline \multirow[b]{2}{*}{ Sl. no. } & \multirow[b]{2}{*}{$\begin{array}{l}\text { Parameter } \\
\text { retrieved }\end{array}$} & \multirow[b]{2}{*}{ Unit } & \multicolumn{3}{|c|}{$\mathrm{ANN}$} & \multicolumn{3}{|c|}{ Bayesian } \\
\hline & & & $\begin{array}{l}\text { Standard } \\
\text { deviation } \\
\text { of } \\
\text { estimate }\end{array}$ & $\begin{array}{c}\text { Correlation } \\
\text { coefficient } \\
(\%)\end{array}$ & $\begin{array}{c}\text { Time } \\
\text { taken } \\
(\mathrm{s})\end{array}$ & $\begin{array}{l}\text { Standard } \\
\text { deviation } \\
\text { of } \\
\text { estimate }\end{array}$ & $\begin{array}{c}\text { Correlation } \\
\text { coefficient } \\
(\%)\end{array}$ & $\begin{array}{c}\text { Time } \\
\text { taken } \\
(\mathrm{s})\end{array}$ \\
\hline 1 & Albedo & - & 0.047 & 98.1 & 0.01 & 0.025 & 99.4 & $\overline{0.03}$ \\
\hline 2 & 1st layer rain & $\mathrm{mm} / \mathrm{h}$ & 4.10 & 95.7 & 0.01 & 2.50 & 98.6 & 0.03 \\
\hline 3 & 2nd layer ice & $\mathrm{mm} / \mathrm{h}$ & 0.41 & 99.7 & 0.01 & 0.01 & 99.9 & 0.03 \\
\hline
\end{tabular}




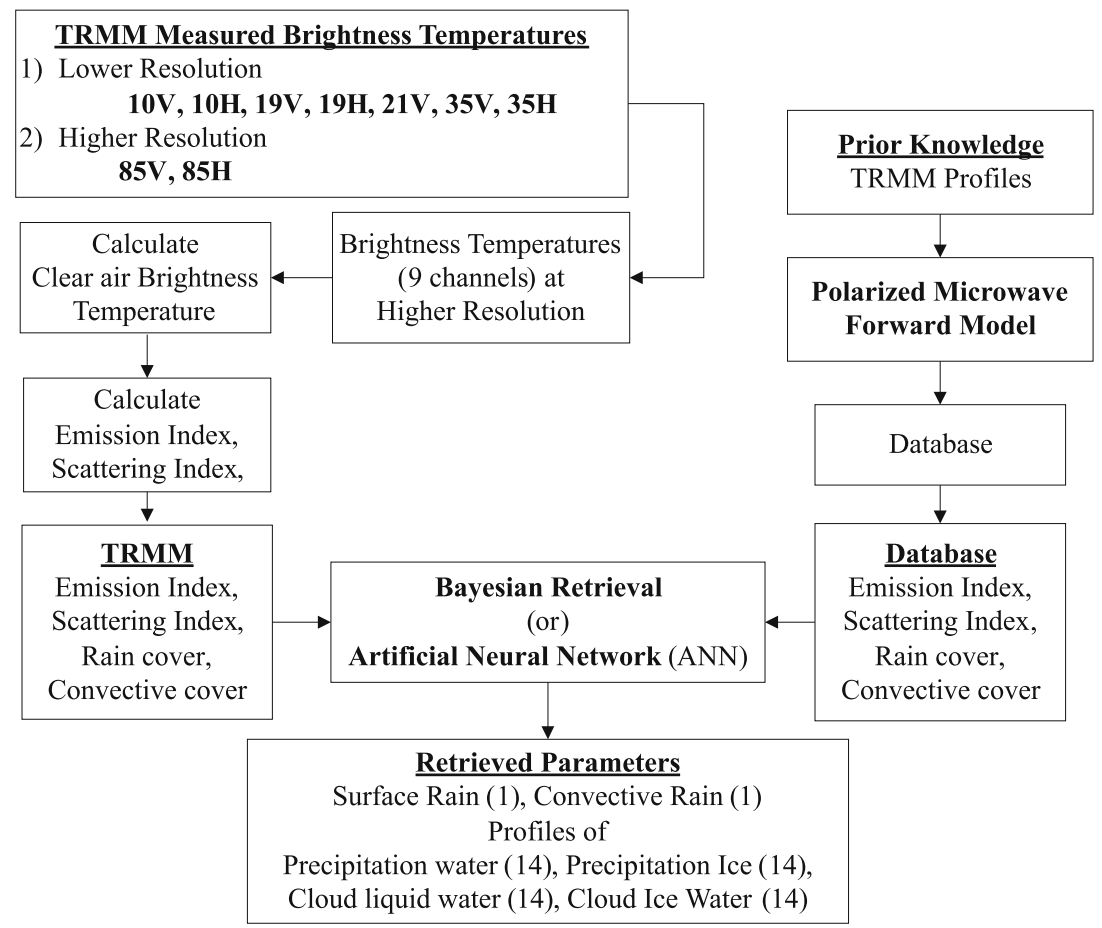

Figure 8. Retrieval algorithm for precipitation related parameters.

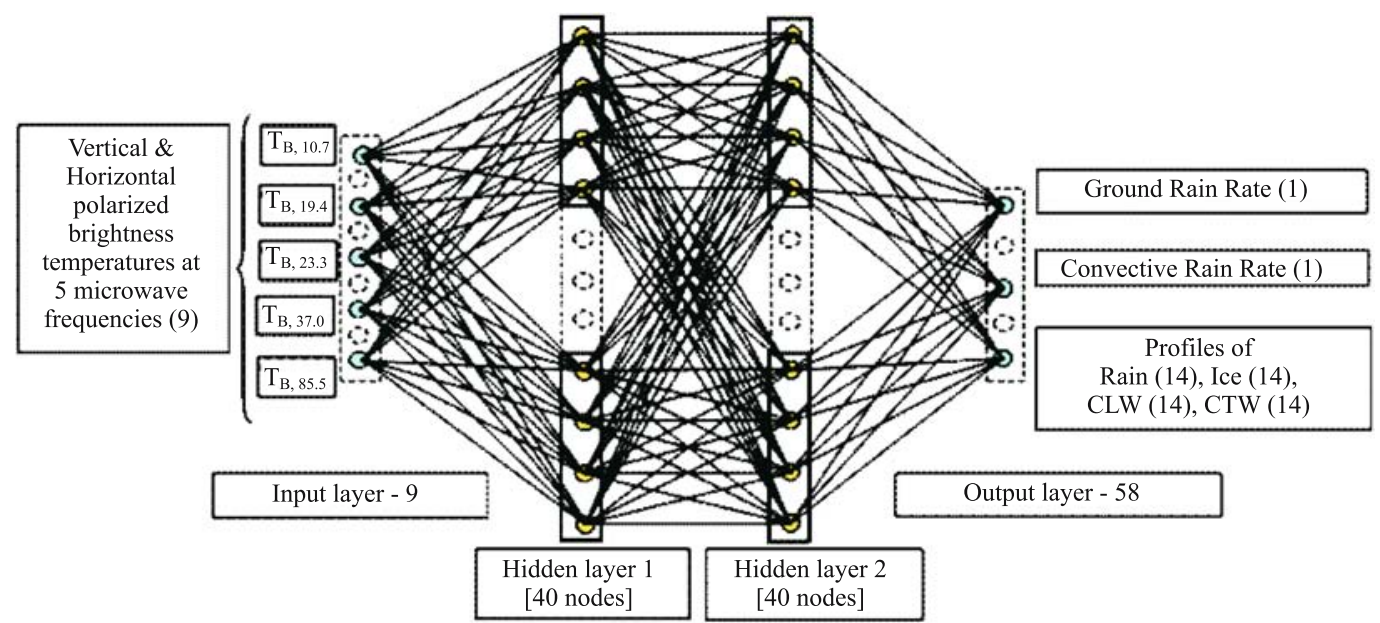

Figure 9. A neural network architecture for precipitation retrievals.

presented here) show that, with no measurement error, both Bayesian and ANN predict very accurately but the accuracy of ANN decreases with increase in the measurement error. A fuller discussion of the retirevals for the two-layer atmosphere are available in Deiveegan (2007).

The above exercise demonstrates that both artificial neural network and Bayesian provide accurate estimation of parameters to be retrieved for a case without measurement error. When noise in the measurements increase, the Bayesian algorithm gives better estimates compared to a neural network. Both these methods give estimation of parameters almost in real time. It can also be observed from the parameter retrievals that the polarized radiative transfer algorithm captures both emission and scattering dominated rain and ice events.

\subsection{The complete retrieval scheme for a 14-layer atmosphere}

A detailed flow diagram elucidating the retrieval procedure for a 14-layer atmosphere is shown in figure 8. The important step of the retrieval algorithm is the creation of database which in a sense 
Table 4. Range of geophysical parameters in the database employed for retrievals.

\begin{tabular}{lllcc}
\hline Sl. no. & Geophysical parameter & Units & Min & Max \\
\hline 1 & Cloud liquid water & $\mathrm{g} / \mathrm{m}^{3}$ & 0 & 0.641 \\
2 & Precipitable water & $\mathrm{g} / \mathrm{m}^{3}$ & 0 & 4.335 \\
3 & Cloud ice & $\mathrm{g} / \mathrm{m}^{3}$ & 0 & 0.372 \\
4 & Precipitable ice & $\mathrm{g} / \mathrm{m}^{3}$ & 0 & 3.474 \\
5 & Ground rain rate & $\mathrm{mm} / \mathrm{h}$ & 0 & 86.7 \\
\hline
\end{tabular}

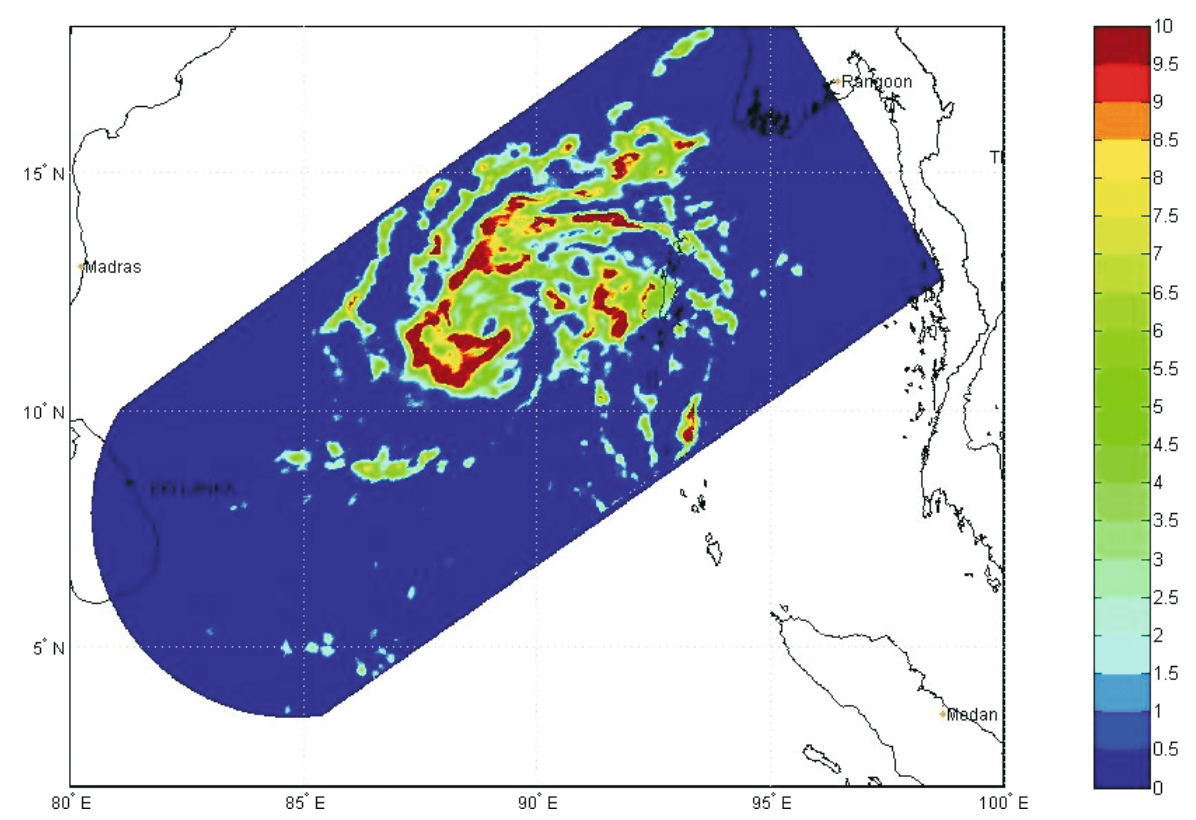

Figure 10. Ground rain rates $(\mathrm{mm} / \mathrm{h})$ retrieved by ANN.

serves as prior knowledge. In the present work, the Tropical Rainfall Measuring Mission (TRMM) retrieved profiles are used as prior knowledge. The TRMM profiles contain the vertical profiles of four hydrometeors (precipitable water, precipitable ice, cloud liquid water and cloud ice) and ground rain rate in a 14-layer plane parallel atmosphere with a total height of $18 \mathrm{~km}$. These are profiles retrieved using the Goddard Profiling Algorithm (GPROF) that powers the TRMM retrievals, using an offline Bayesian. The GPROF uses the vertical profiles of hydrometeors numerically generated with high resolution numerical weather prediction models and these profiles are commonly referred to as the Goddard Cloud Ensemble (GCE) profiles (see, for example, Kummerow et al 1996).

In the present study, TRMM retrieved profiles of earlier cyclones that have occurred either in the Bay of Bengal or the Arabian Sea are directly given as input to the polarized forward model. Details of the database of profiles employed in the retrievals are discussed in section 3.1.

The forward model solves the equation of transfer, given the vertical profiles of hydrometeors.
An SST of $300 \mathrm{~K}$, wind speed appropriate to the strength of the storm and a salinity of $36 \mathrm{PPM}$ have been used in the calculations. The pressure, temperature and humidity profiles have been taken from the Goddard Cloud Ensemble (GCE) Profiles for a raining atmosphere. Preliminary studies showed that a variation of $\pm 2 \mathrm{~K}$ in the SST from the baseline value of $300 \mathrm{~K}$ did not produce any significant change in the output of the forward model under raining conditions. This is because of the domination by emission and scattering of the hydrometeors in a precipitating atmosphere. The output of the forward model is the top of the atmospheric (TOA) radiances at various frequencies. The database contains the profiles of hydrometeors and corresponding brightness temperatures at TRMM frequencies (vertical and horizontal polarization for 10.65, 19.35, 37, $85.5 \mathrm{GHz}$ and vertical polarization for $21.3 \mathrm{GHz}$ ). The brightness temperatures are converted into non-dimensional parameters called emission and scattering indices. These indices are dimensionless numbers. They are the ratios of the difference between vertical and horizontal polarization 


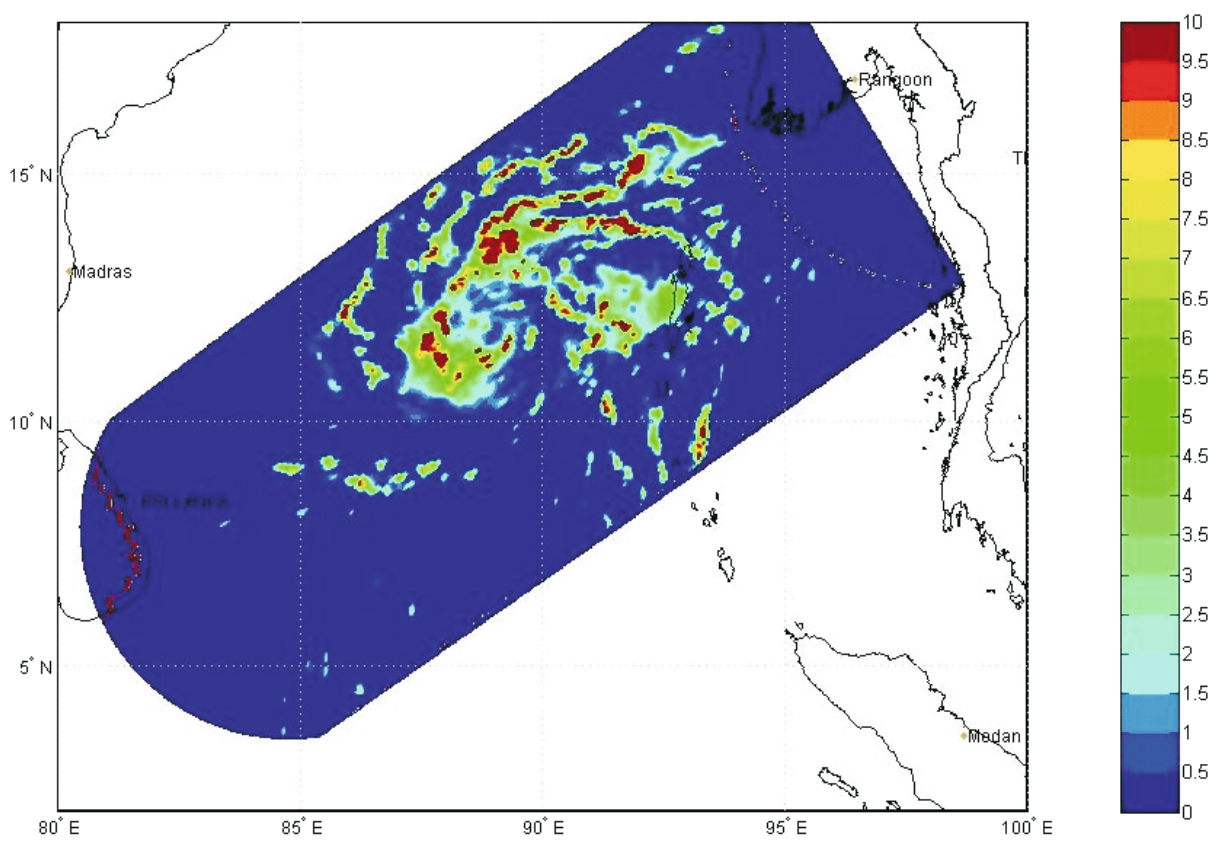

Figure 11. Ground rain rates $(\mathrm{mm} / \mathrm{h})$ retrieved by Bayesian.

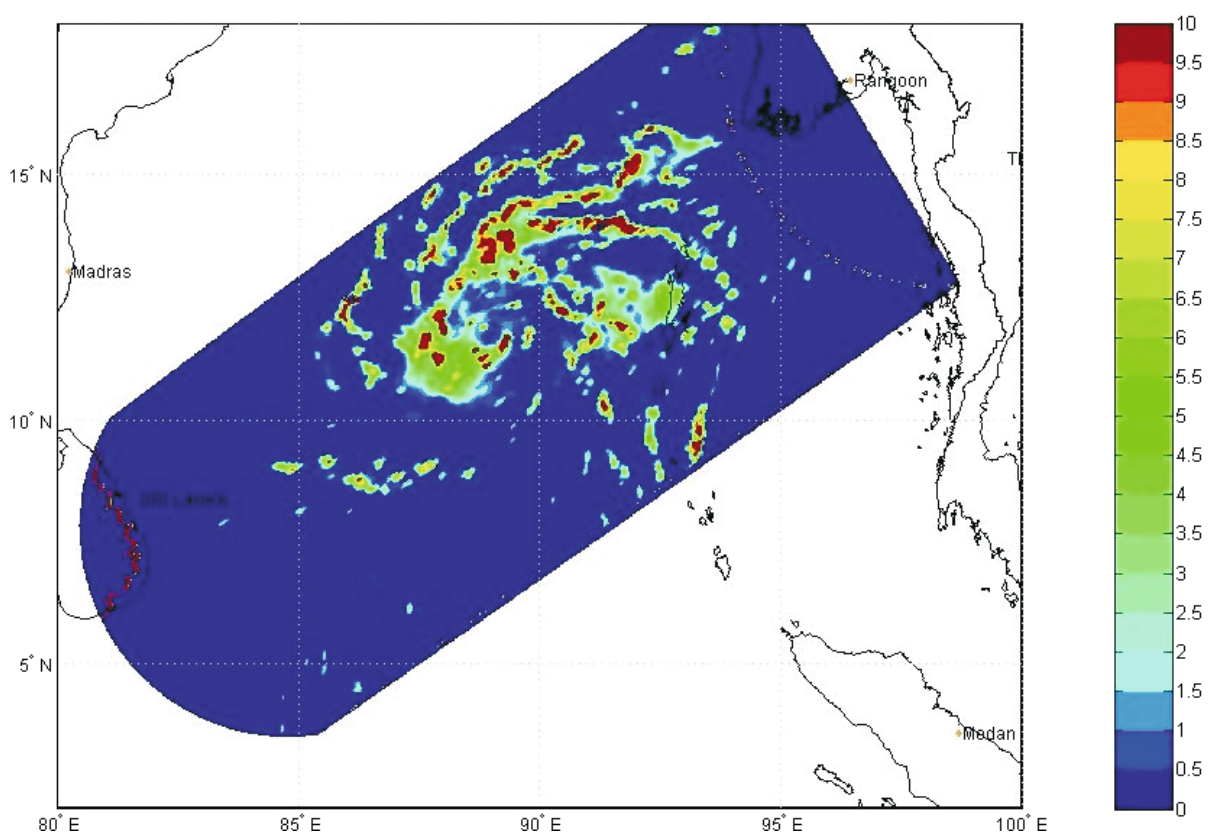

Figure 12. Ground rain rates $(\mathrm{mm} / \mathrm{h})$ retrieved by TMI algorithm.

brightness temperatures normalized by the difference between the same two quantities but for clear sky conditions. This has several advantages like reducing roundoff errors and the dimensionality. More importantly, by employing this procedure, any bias induced by the radiometer or the forward model is also more or less nullified (see Petty 2001 for a fuller discussion on this). The satellite radiometer measures the brightness temperatures at different frequencies and polarizations. Furthermore, the TRMM brightness temperatures for different frequencies are available at different resolutions. First, the retrieval algorithm converts these data into high resolution data. The brightness temperatures are then converted into emission and scattering indices. The retrieval algorithm compares the non-dimensional brightness temperatures obtained from simulations and the TRMM measurements. The retrieval algorithm then gives the retrieved parameters 


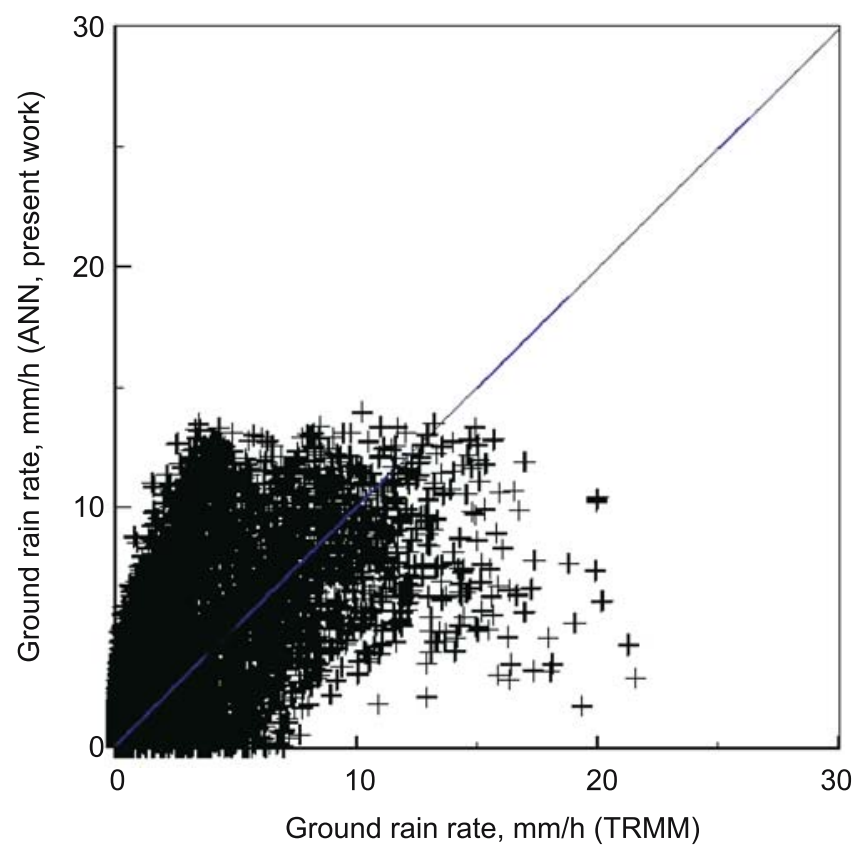

Figure 13. Parity plot of ground rain rate retrieved by ANN with TMI algorithm.

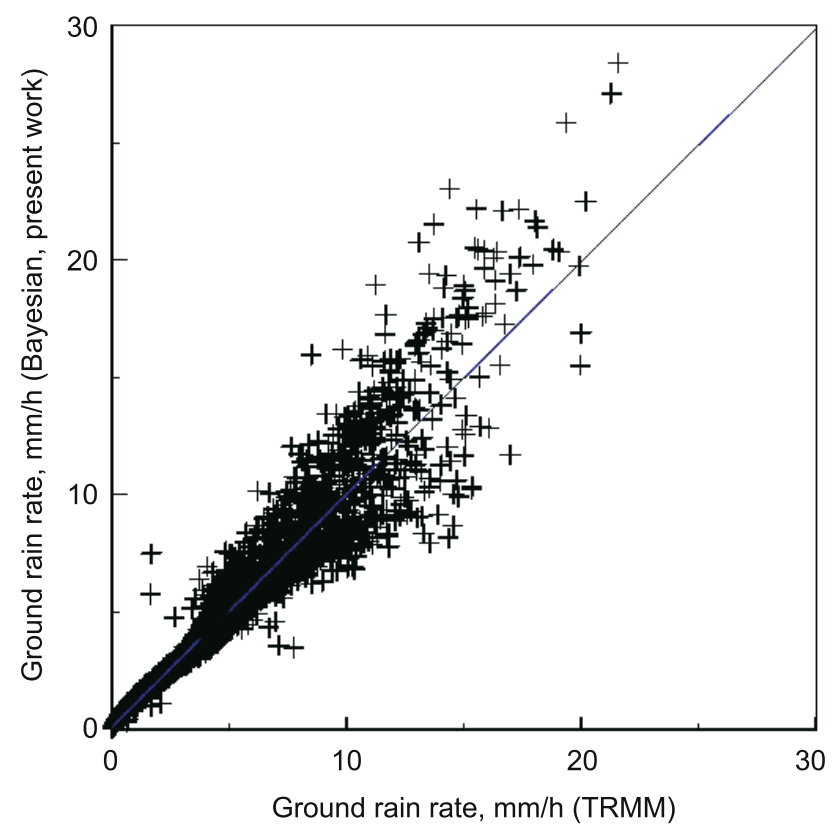

Figure 14. Parity plot of ground rain rate retrieved by Bayesian with TMI algorithm.

by minimizing the differences between these two vectors.

For brevity, details concerning the calculation of the non-dimensional brightness temperatures from actual measured brightness temperatures and the screening algorithms used in the present work (to eliminate the nonraining pixels and pixels over land) are not presented here. See Deiveegan et al (2008) for a fuller discussion on this.
In so far as ANN retrievals are concerned, feed-forward multilayer perceptron networks are employed for training the ANN. These networks are widely used due to their simplicity and excellent performance (Peterson et al 1994). A network with 9 inputs and 58 outputs (56 hydrometeors, ground rain rate and convective fraction) is created, and two hidden layers with 40 neurons each are employed. Figure 9 shows the layout of the network used in the present study. The left most layer in the figure corresponds to the input variables. All the input variables are normalized with respect to their maximum possible values, to ensure that the range of the entire variables lie in the same range of activation function used for each node in the neural network. This helps in reducing the effort to link the input and output values in the ANN. Furthermore, this also reduces some effort needed to code the variable limits in the optimization analysis used for accomplishing the retrievals. The number of hidden layers used is two, and 40 nodes are placed in each hidden layer. The rightmost nodes in the network give the retrieval parameters corresponding to the input brightness temperatures at various channels. The above configuration of the network is selected based on the performance of the network that predicts the parameters well as opposed to other configurations.

\section{Application to a tropical storm: A case study}

Accurate quantitative assessment of rainfall and hydrometeor profiles within tropical cyclones over oceans is a difficult problem because direct measurements are almost impossible. The ANN and the Bayesian retrieval algorithms, described in the previous section, are tested for tropical cyclonic storms. The specific storm under consideration is FANOOS that made its landfall on the southeast coast of India on 9 December 2005. The satellite (TRMM) measured brightness temperatures at $10.65,19.35,21.3,37,85.5$ (2 polarizations each except for the $21.3 \mathrm{GHz}$ channel that does not employ the horizontal polarization) are given as input to the retrieval algorithms. The retrieval algorithms discussed in section 2 are used to estimate the profiles of rain, cloud liquid water, cloud ice, precipitation ice, convective rain rate and ground rain rate. The retrieved parameters are compared with the TRMM retrieved vertical profiles of hydrometeors. Additionally, the retrieval is validated with rain rate obtained from the precipitation radar, which is considered as proxy evidence. 

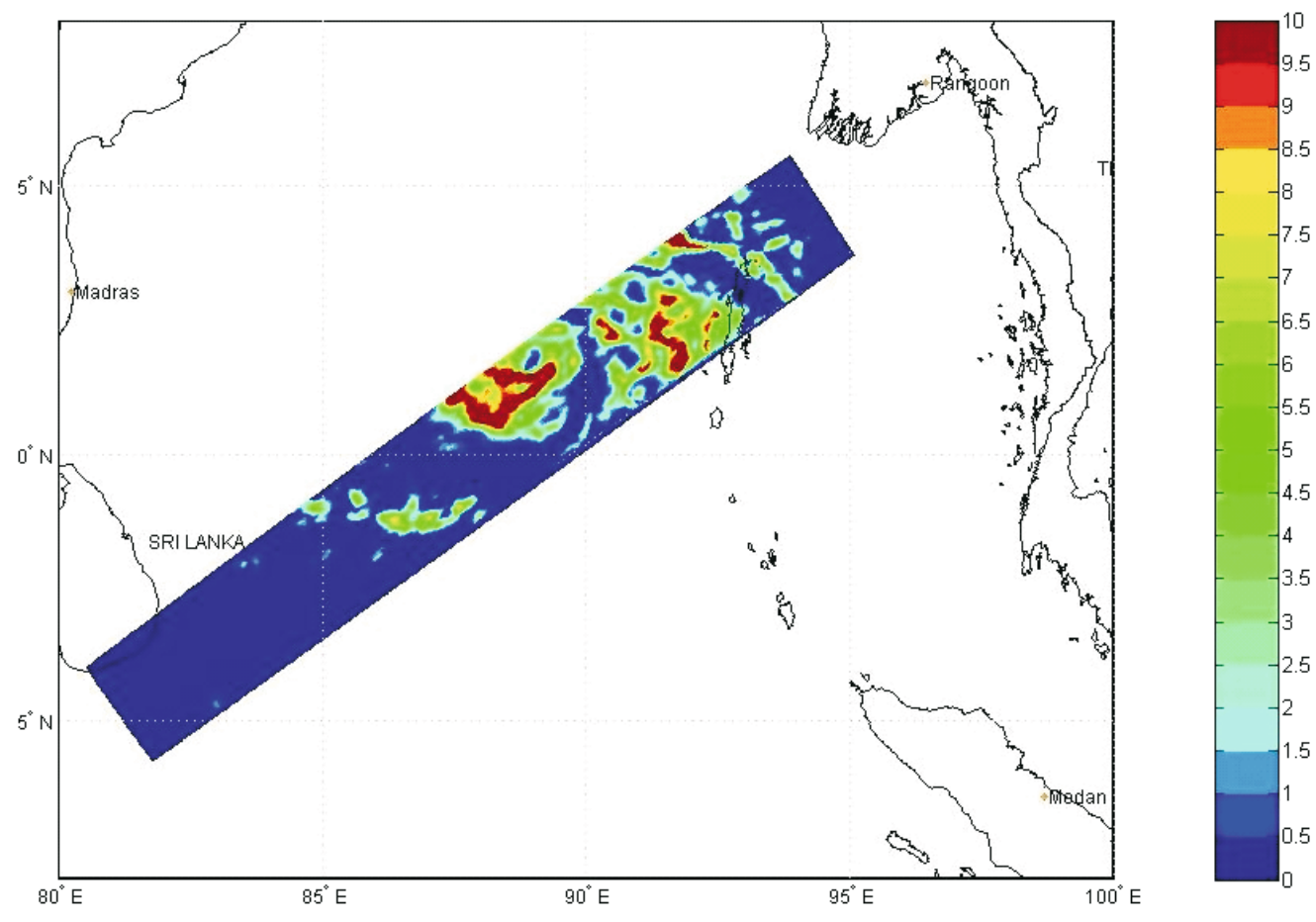

Figure 15. Ground rain rates retrieved by ANN.

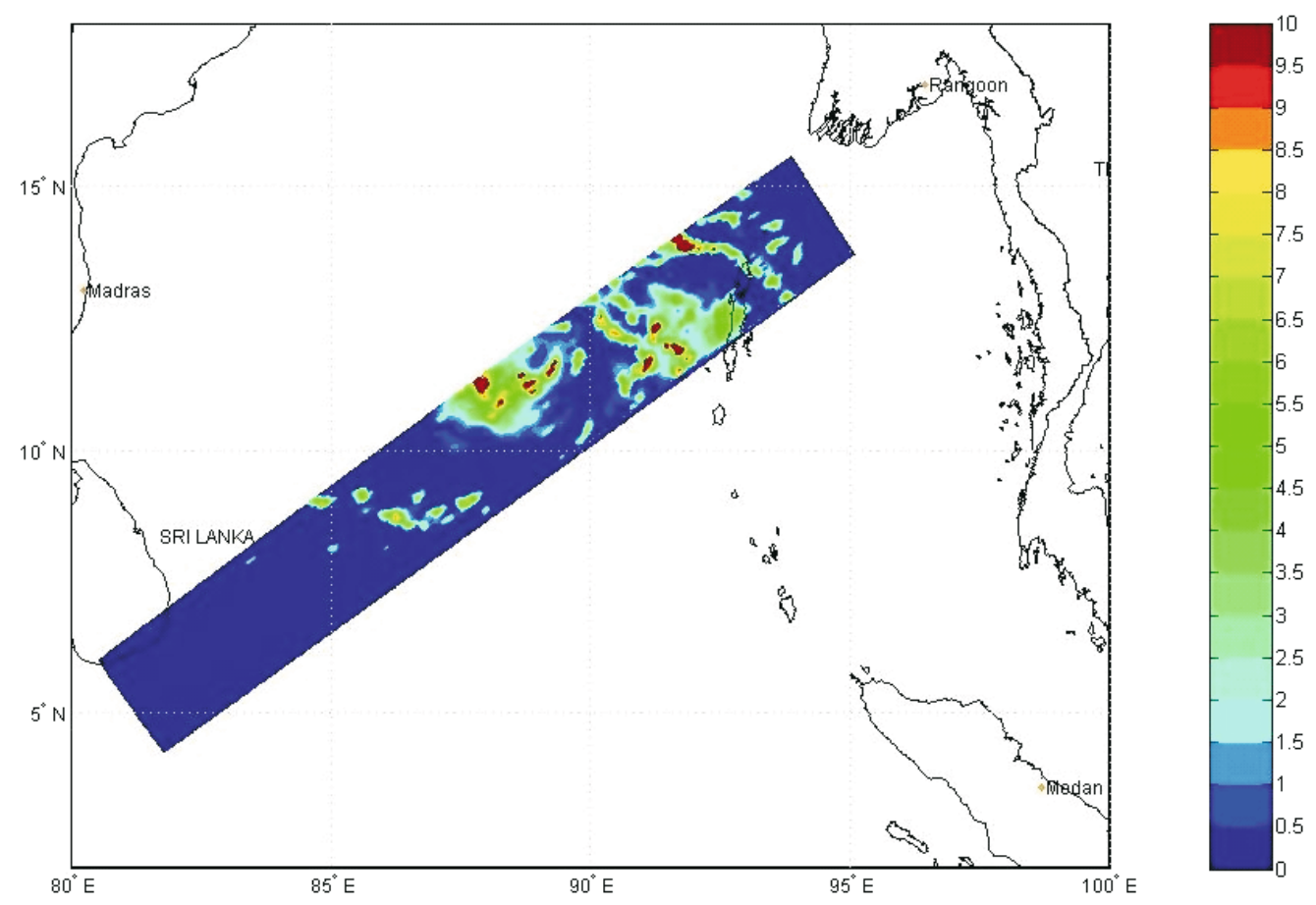

Figure 16. Ground rain rates retrieved by Bayesian.

\subsection{Generation of database}

In the present study, the objective is to evaluate the accuracy and efficiency of the retrieval algorithm to retrieve an extreme event like a tropical cyclone. The Bayesian algorithm and ANN used in this study are both data driven approaches and hence the generation of database holds the key for both the methods. The distribution of the hydrometeor parameters is the prior information in the retrieval, so it is important that the profiles are realistic and completely cover the possible realistic 


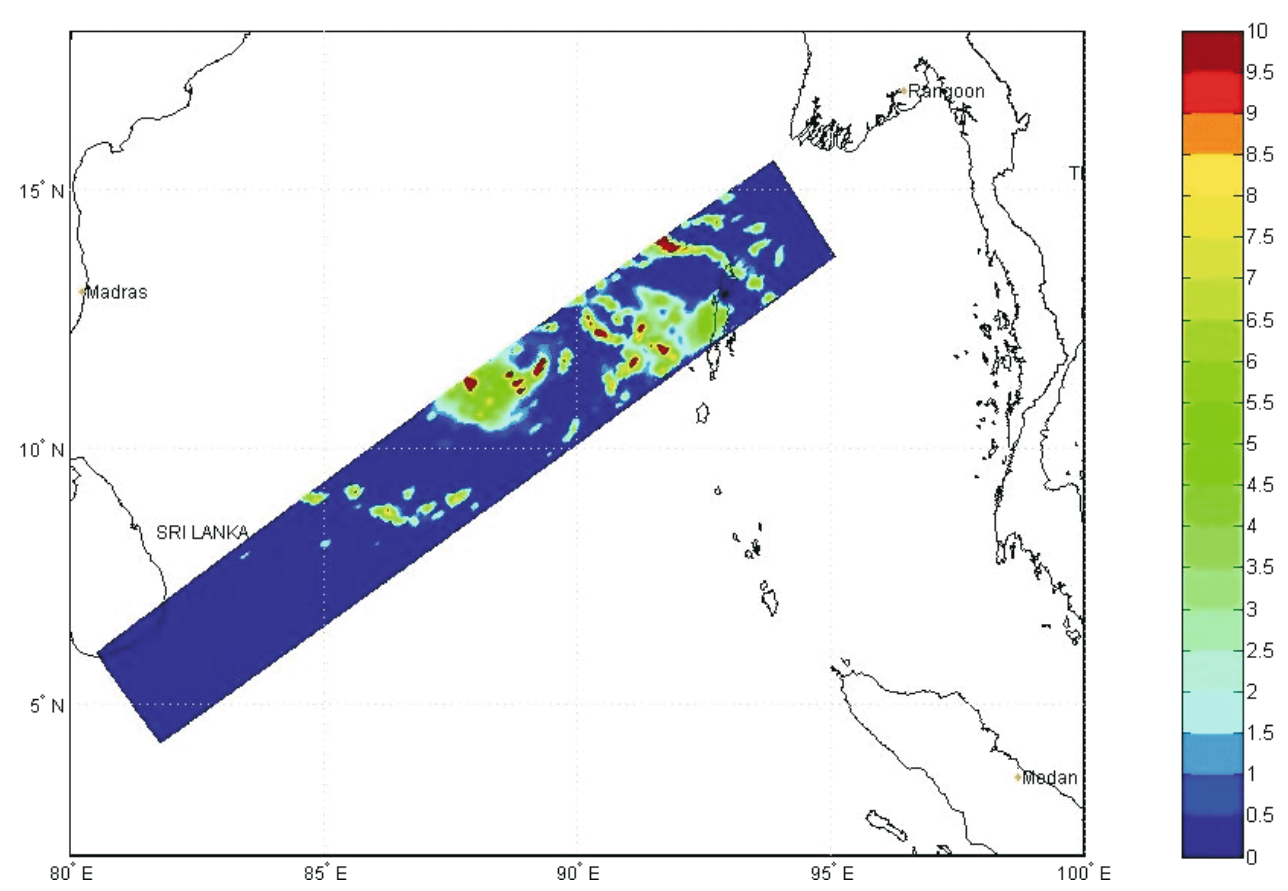

Figure 17. Ground rain rates retrieved by TMI algorithm (Bayesian).

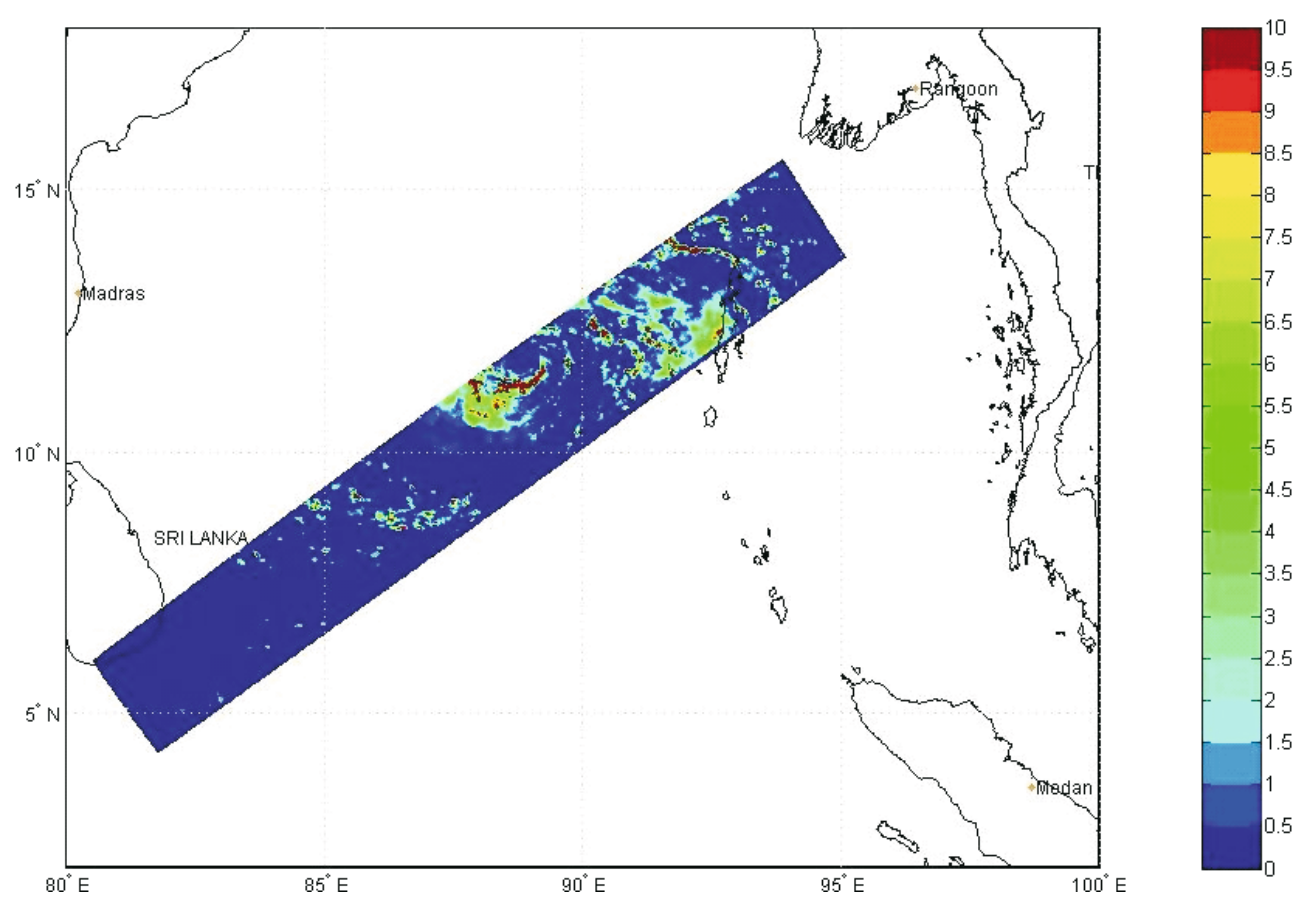

Figure 18. Ground rain rates measured by precipitation radar (PR).

parameter range. There are two parts in generating the database:

(1) creating the profiles of rain rates, cloud liquid water content, cloud ice and precipitation ice,

(2) computing the brightness temperatures at different frequencies and polarization.
Towards this, a retrieval database was first created by considering five tropical cyclones over the Indian subcontinent that occurred between years 2000 and 2004. Approximately 43,000 profiles and the corresponding brightness temperatures are stored in the database. Some statistics of the database are shown in table 4 . The retrieval algorithms are then applied to the tropical cyclone 


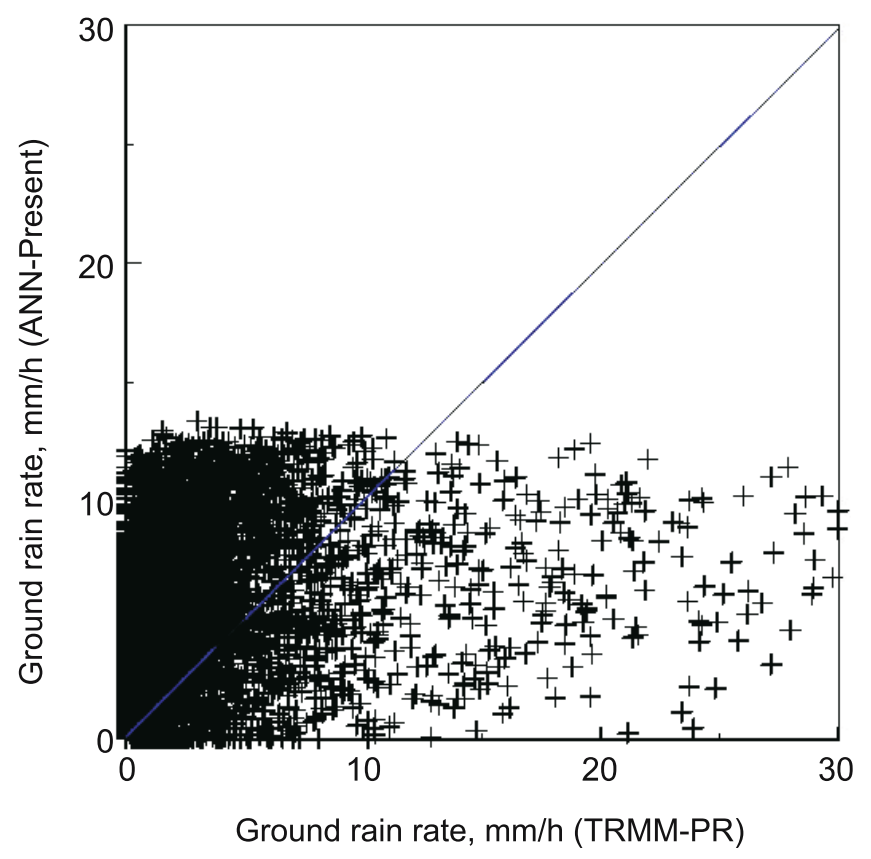

Figure 19. Parity plot of ground rain rates retrieved by ANN with PR.

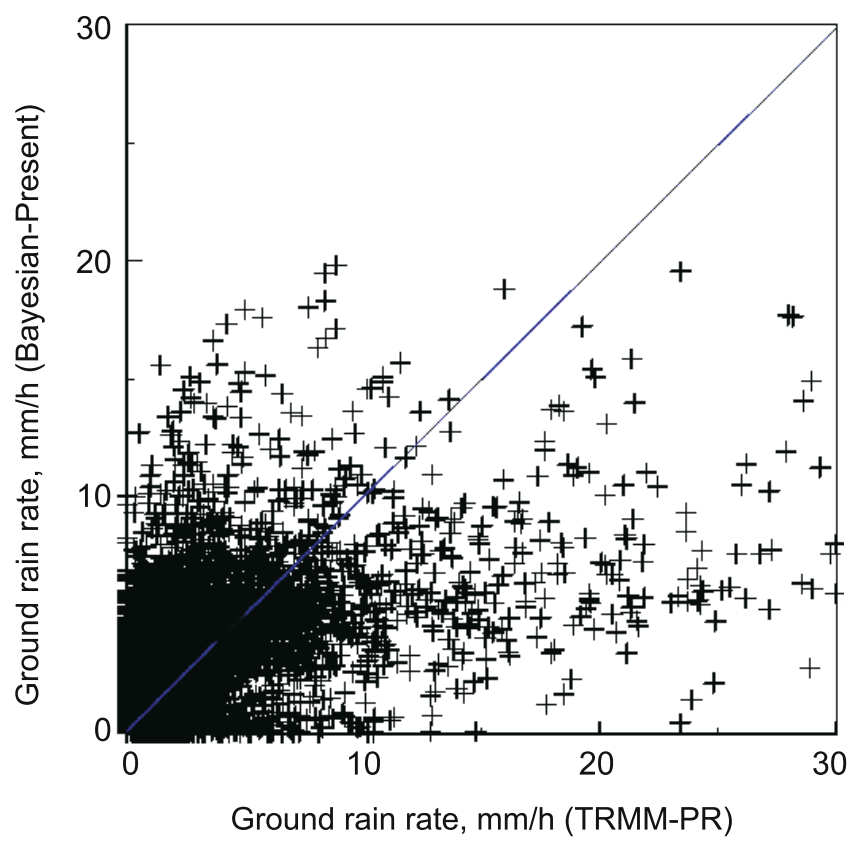

Figure 20. Parity plot of ground rain rate retrieved by Bayesian with PR.

FANOOS and as a final validation exercise for cyclone 03B.

\subsection{Results for the case study}

Figures 10-12 show the surface rain rate retrieved by ANN, Bayesian and TMI (TRMM microwave imager) retrieval algorithms, respectively for the tropical cyclone FANOOS. The comparison with

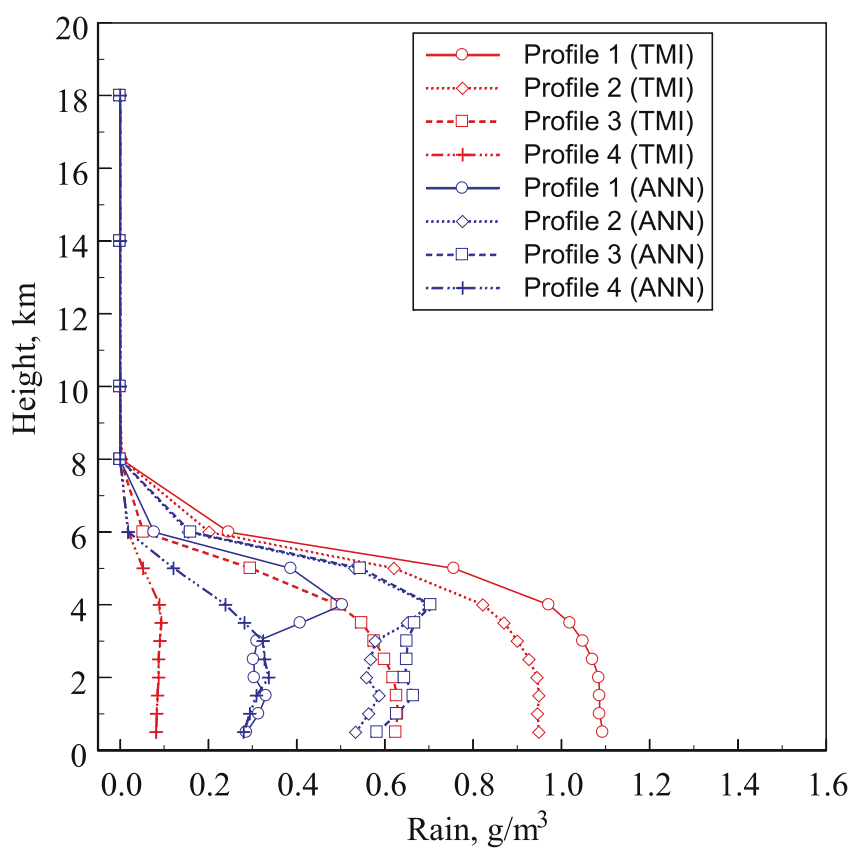

Figure 21. ANN retrieved vertical profiles of rain.

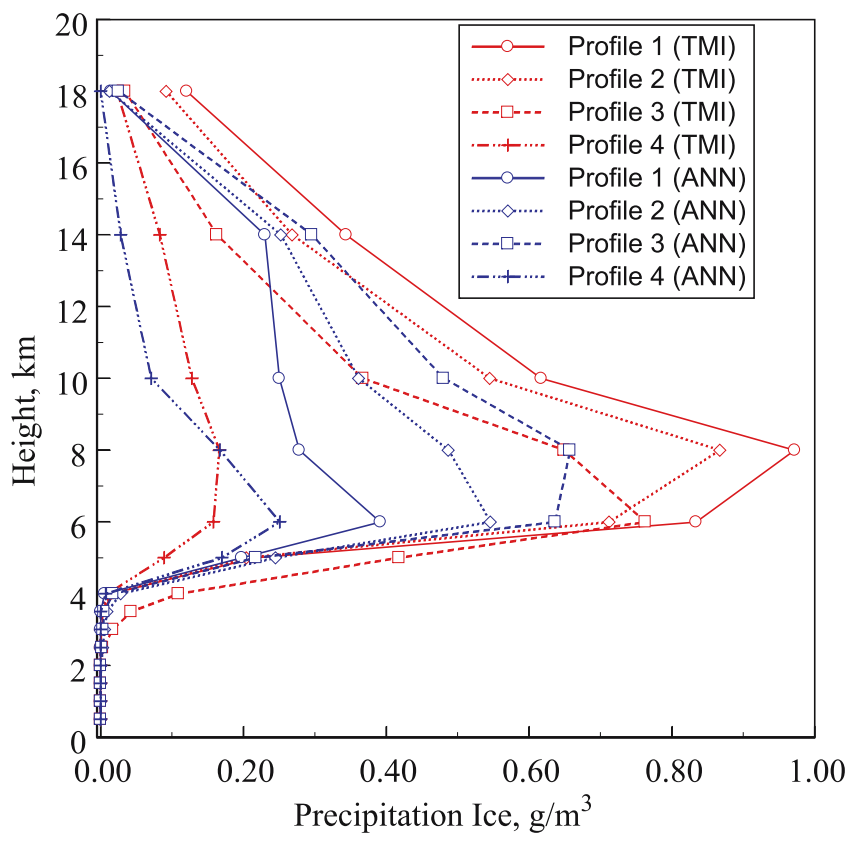

Figure 22. ANN retrieved vertical profiles of precipitating ice.

TMI retrievals shows that the two algorithms, in general give a good estimation of ground rain rate. Both ANN and Bayesian algorithms are able to capture the general features of the cyclone quite well. Particularly, the Bayesian retrieved ground rain rate closely matches with the TMI retrieved ground rain rate (the high precipitation over Sri Lanka seen in figures 11 and 12 may be ignored as it is an artifact of the interpolation. This work concerns retrievals only over the ocean). 


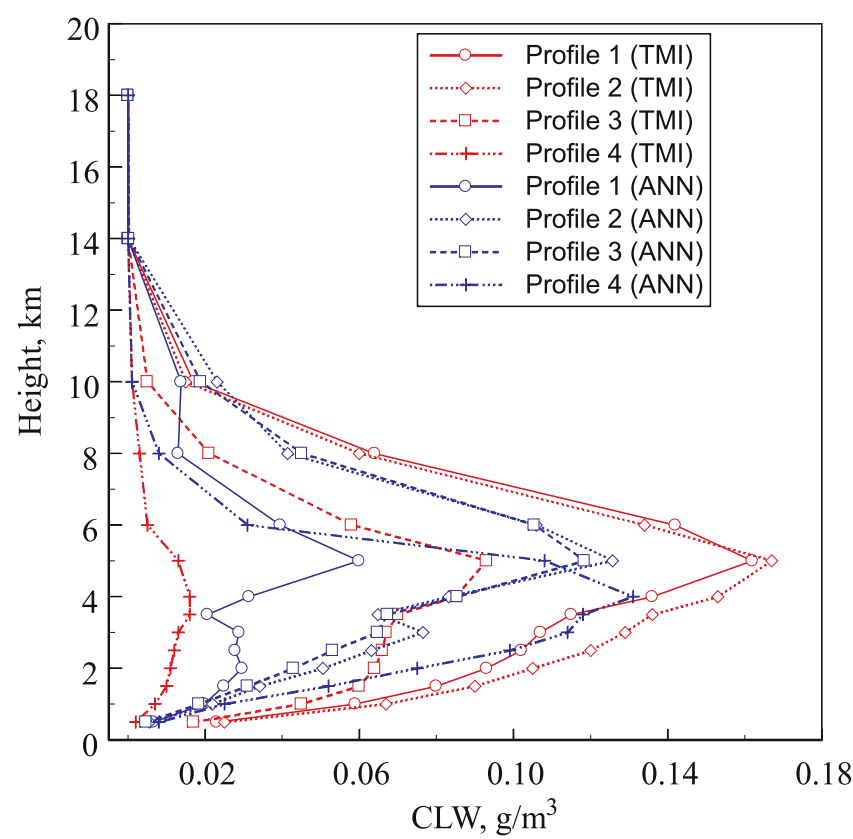

Figure 23. ANN retrieved vertical profiles of cloud liquid water.

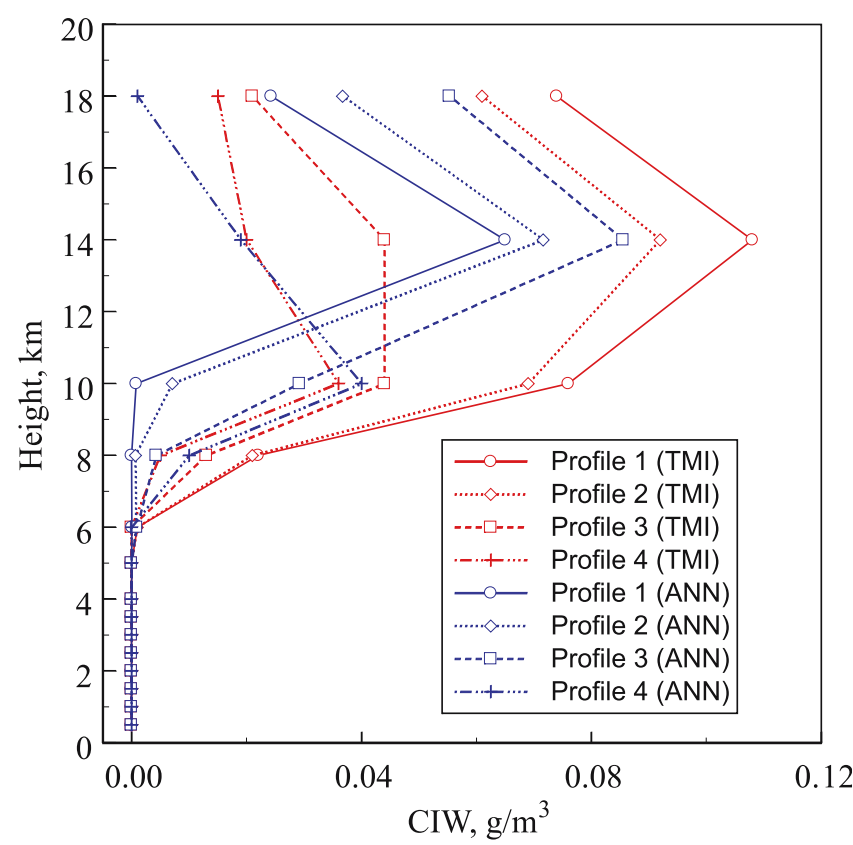

Figure 24. ANN retrieved profiles of cloud ice.

Figures 13 and 14 show parity plots of surface rain rate retrieved by the ANN and Bayesian with the surface rain rate retrieved by the TMI. The superior performance of the Bayesian method when compared to ANN can be inferred from these figures. The high dimensionality of the problem wherein 58 parameters are retrieved from 9 measurements, makes the ANN retrieval less accurate. However, it cannot be denied that the ANN retrievals are fast and easy to implement.

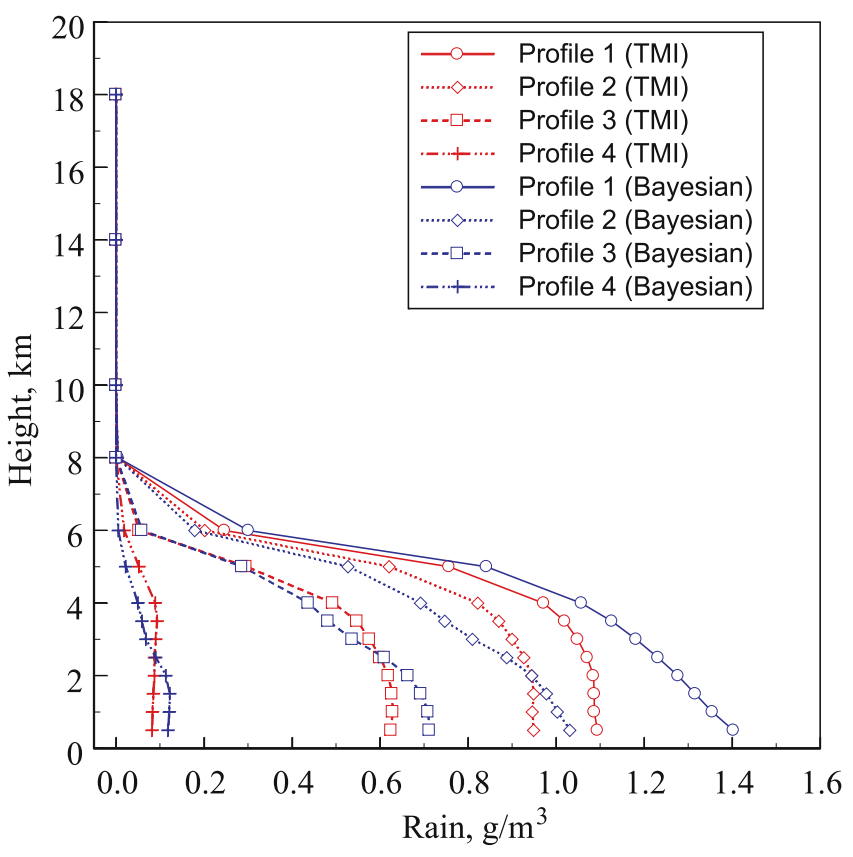

Figure 25. Bayesian retrieved profiles of rain.

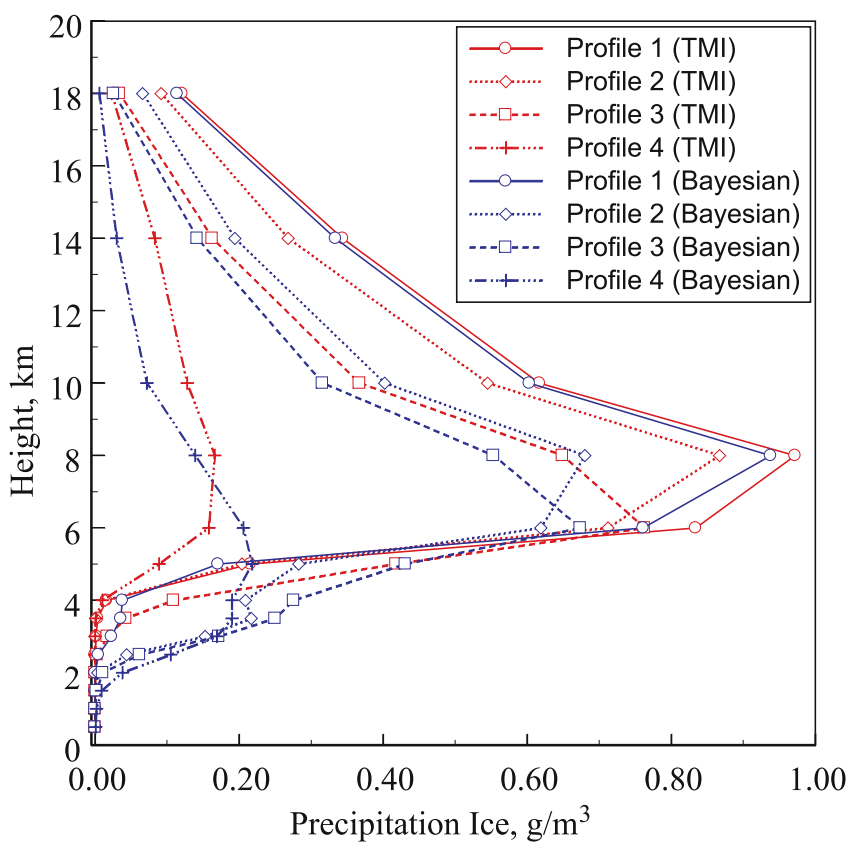

Figure 26. Bayesian retrieved profiles of precipitating ice.

Figures 15 and 16 show the surface rain rate retrieved by the ANN and Bayesian algorithms used in the present work. These retrievals are compared with the TMI retrieved (figure 17) and precipitation radar (PR) (figure 18) estimated ground rain rates. Additionally, parity plots between the ground rain rate obtained from present study (ANN, Bayesian) and the PR data are shown in figures 19 and 20.

The general agreement between PR data and retrieved rain rates is reasonably good. The general 


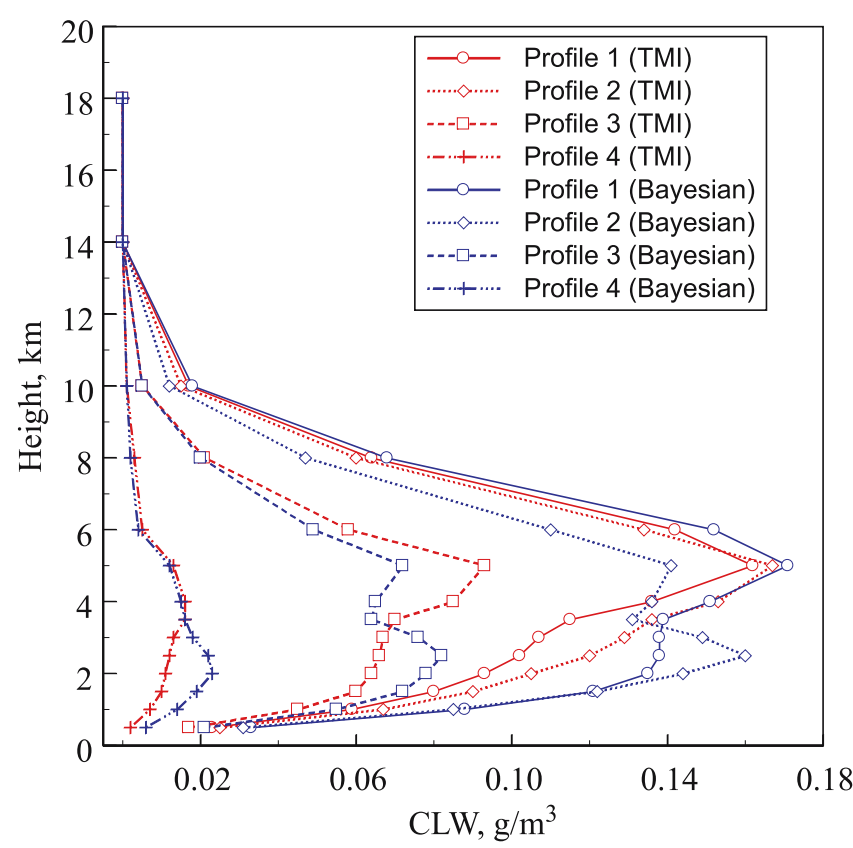

Figure 27. Bayesian retrieved profiles of cloud liquid water.

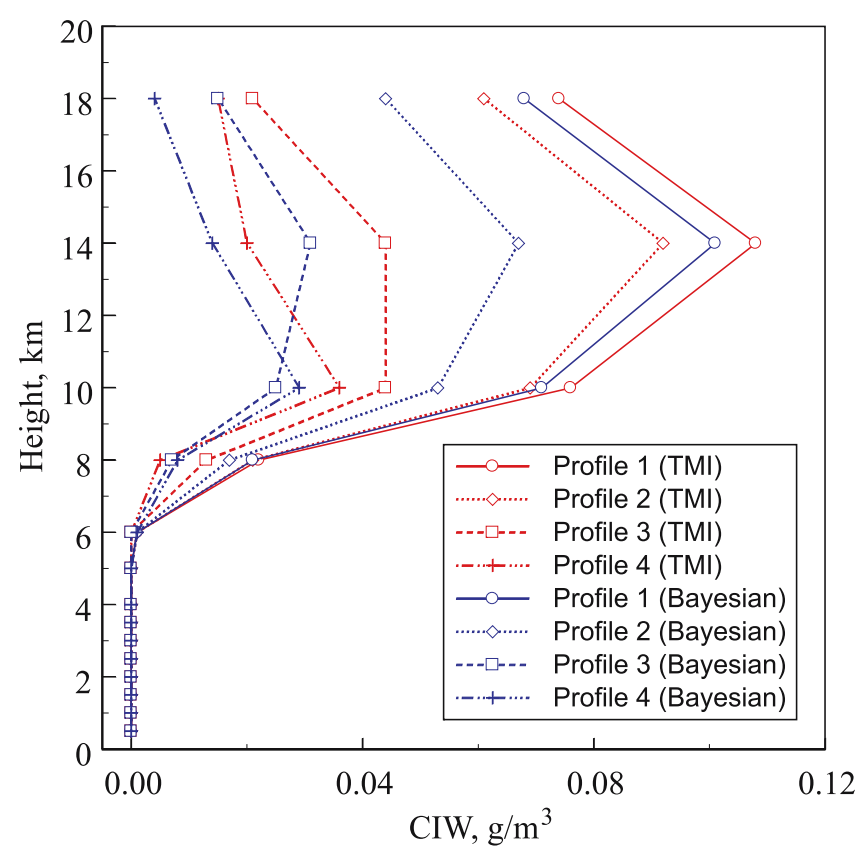

Figure 28. Bayesian retrieved profiles of cloud ice.

pattern of tropical storm is very well reproduced. The correlation coefficient between the PR data and surface rain rate retrieved by the ANN, Bayesian and TMI algorithms are 0.474, 0.572 and 0.596 with standard deviations $(\mathrm{mm} / \mathrm{h})$ of 3.101 , 2.603 and 2.472, respectively. From the above it is seen that the present Bayesian algorithm is almost as good as the TMI algorithm as both of them have a correlation coefficient of around $60 \%$. At heavier rain rates, uncertainties significantly increase due to heavy attenuation in all channels which requires surface rainfall to be inferred as the sole relationship (non-unique) between surface rain rate and scattering signature in the strongly coupled 37 and $85 \mathrm{GHz}$ brightness temperatures. The TMI retrieval is considered to be the stateof-the-art and the Bayesian retrieval developed in this study is very close to the TMI retrieval in terms of accuracy even though the forward model that drives the retrieval does not address (i) the effect of the melting layer and (ii) the beam filling problem.

Figures 21-24 show the ANN retrieved profiles of rain, precipitation ice, cloud liquid water and cloud ice, for three representative rain profiles that correspond to low, medium and high rain rate. The retrieved profiles are, in general, physically consistent despite the high dimensionality of the problem and the common perception that retrieved vertical profiles are 'quite noisy'. The above statement is substantiated by the fact that the cloud ice content is close to zero in up to the bright band, cloud liquid water tapers off at an altitude of $10 \mathrm{~km}$, precipitation ice peaks at around $8 \mathrm{~km}$ and cloud ice peaks around $14 \mathrm{~km}$. Additionally, the profiles are compared with TRMM-TMI retrieved profiles. The comparison shows that the ANN retrieved profiles are not in very good agreement with TMI retrieved profiles. This is due to the fact that ANN uses equal weights for all the channels. However, the Bayesian algorithm gives maximum weight for the channels which give more information about precipitation, i.e., higher frequency channels. Figures 25-28 show a comparison of the vertical profiles obtained from the present Bayesian algorithm with the TMI retrieved data. It can be observed that the present Bayesian retrievals are in very good agreement with TMI retrievals.

As a final validation exercise, retrievals were also done for cyclone 03B that again originated in the Bay of Bengal during the final week of June 2007, a rather unusual period for cyclone activity in this region. The cyclonic activity in the Bay is invariably present only during the period of the north-east monsoon, i.e., during October-December. In view of this, artifacts, if any, introduced by our retrieval procedure will clearly reflect in the quality of the retrievals, if the algorithm is tested for an unusual cyclone like the 03B. Furthermore, to remove the influence of any bias or prejudice introduced by screening for rain pixels, retrievals were performed for 03B by switching off the rain flags. Figures $29(\mathrm{a}-\mathrm{c})$ show the retrieval of the ground rain rate with and without rain screening, along with the TMI retrievals. The performance of the retrieval algorithm is (i) quite impressive and (ii) more or less unaffected by the rain screening, both of which reinforce our faith in the retrieval process. 

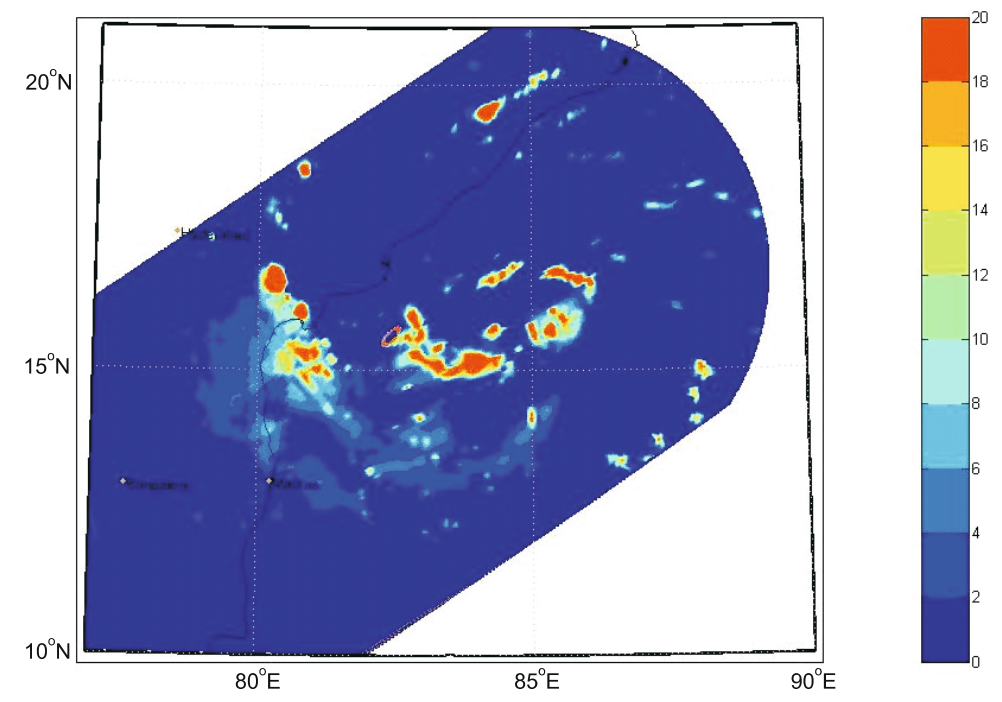

Figure 29(a). Ground rain rate retrievals for cyclone 03B (TRMM).
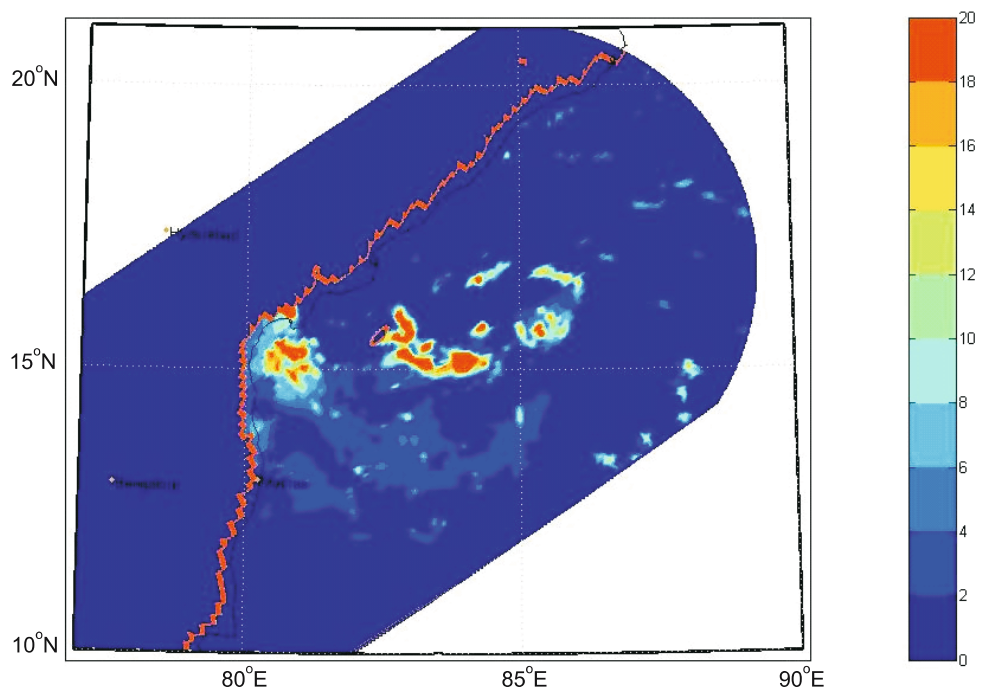

Figure 29(b). Ground rain rate retrievals for cyclone 03B with Bayesian using rain/no rain classification.
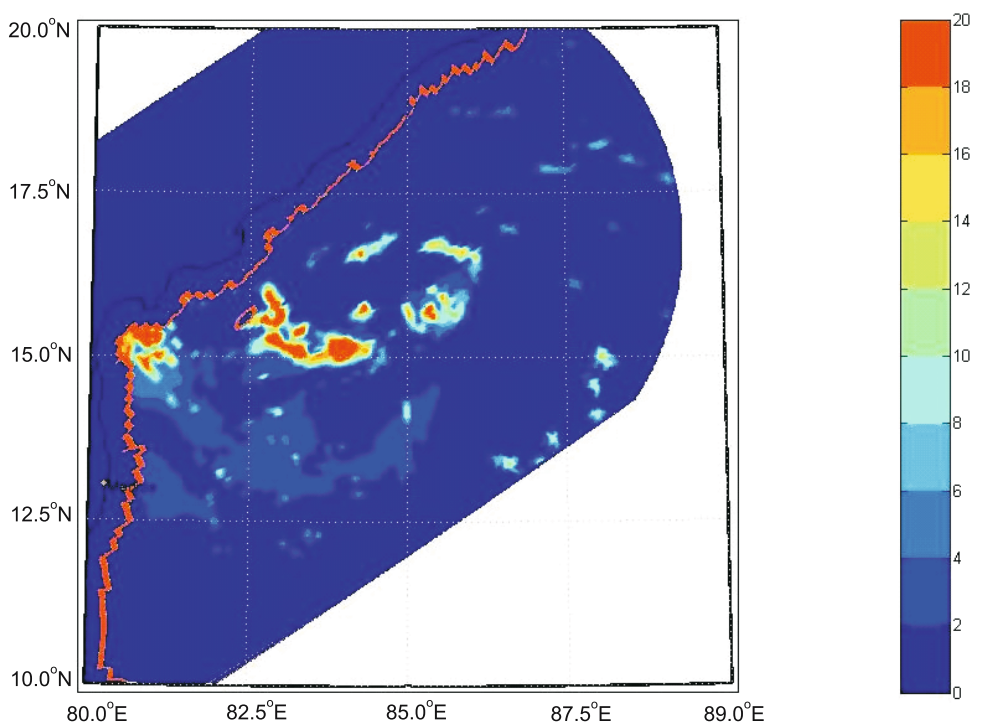

Figure 29(c). Ground rain rate retrievals for cyclone 03B with Bayesian without using rain/no rain classification. 


\section{Conclusions}

This paper discussed two data driven techniques namely Bayesian and ANN for estimating rainfall and hydrometeors from (i) synthetically generated radiances from a two-layer atmosphere and (ii) satellite microwave radiances at frequencies corresponding to TRMM. The retrievals are powered by a polarized microwave radiative transfer code developed in-house. The ANN is easy to implement, and despite the high dimensionality of the problem, gave results that are reasonable but still not close to the state-of-the-art. On the contrary, the Bayesian performed quite well with accuracy very close to the state-of-the-art.

The key novelties of the present work are: (i) use of polarization from both the oceans and the hydrometeors and (ii) populating the database with already retrieved profiles, for similar situations in the past. If retrievals are to be done for a tropical cyclone, we start with a database of retrieved profiles of earlier cyclones. Since any retrieval minimizes the error between satellite observed and simulated radiances, injecting 'hard' data from measurements by employing retrieved profiles makes the retrieval better. We demonstrated this methodology first for the tropical cyclone FANOOS and then for the cyclone 03B both of which originated in the Bay of Bengal. The agreement with the gold standard radar estimates (PR estimates) is close to $60 \%$ and is close to that obtained by the GPROF, in spite of the melting layer not taken into account and the beam filling problem. However, it needs to be mentioned that both the ANN and Bayesian algorithms are data driven and extensive training holds the key to successful retrievals. With inherent noise in the data, the Bayesian is more robust.

Hence, the strategy of doing offline Bayesian retrievals with a dynamically updated database that incorporates data corresponding to similar rain events in the past, from the satellite under consideration, for future retrievals from the same satellite holds considerable promise and warrants further investigations.

\section{References}

Deiveegan M 2007 Precipitation retrieval using a polarized microwave radiative transport model, Ph.D. Thesis, Department of Mechanical Engineering, Indian Institute of Technology Madras, India.

Deiveegan M, Balaji C and Venkateshan S P 2008 A polarized microwave radiative transfer model for passive remote sensing; Atmos. Res. 88 277-293.

Evans K F and Stephens G L 1991 A new polarized atmospheric radiative transfer model; J. Quantitative Spectroscopy and Radiative Transfer 46 413-423.

Hagan M T and Menhaj M B 1994 Training feed forward nets with the Marquardt algorithm; IEEE Transactions on Neural Network 5 989-993.

Kummerow C, Olson W S and Giglio L 1996 A simplified scheme for obtaining precipitation and vertical hydrometeor profiles from passive microwave sensors; IEEE Transactions on Geosciences and Remote Sensing 34 $1213-1232$.

Lippmann R P 1987 An introduction to computing with neural nets; IEEE ASSP Magazine 4 4-22.

Marquardt D W 1963 An algorithm for least-squares estimation of nonlinear parameters; J. Soc. Industrial and Applied Mathematics 11 431-441.

Marzano F S, Mugnai A, Panegrossi G, Pierdicca N, Smith E A and Turk J 1999 Bayesian estimation of precipitating cloud parameters from combined measurements of space borne microwave radiometer and radar; IEEE Transactions on Geoscience and Remote Sensing 37 596-613.

Nguyen D and Widrow B 1990 Improving learning speed of 2-layer neural networks by choosing initial values of the adaptive weights; International Joint Conference of Neural Networks 3 21-26.

Peterson C, Roghvaldsson T and Lonnblad L 1994 JETNET 3.0, A versatile artificial neural network package; Computer Physics Communications 81 185-220.

Petty W G 2001 Physical and Microwave Radiative Properties of Precipitating Clouds. Part I: Principal Component Analysis of Observed Multichannel Microwave Radiances in Tropical Stratiform Rainfall; J. Appl. Meteorol. 40(12) 2105-2114.

Srinivasan J 2006 MEGHA TROPIQUES Scientific Rationale, First Workshop on Megha-Tropiques organized by Indian Space Research Organization (ISRO), Indian Institute of Science (IISc), Bangalore. 